\title{
Macrophage-derived exosomal miR-342-3p promotes progression of renal cell carcinoma through the NEDD4L/CEP55 axis-mediated $\mathrm{PIBK} / \mathrm{AKT} / \mathrm{mTOR}$ signaling pathway
}

Jia-fu Feng ( $\square$ fengjiafu@uestc.edu.cn )

Mianyang Central Hospital, University of Electronic Science and Technology of China

Bei Xu

Mianyang Central Hospital, University of Electronic Science and Technology of China chunmei dai

Mianyang Central Hospital, University of Electronic Science and Technology of China Yao-dong Wang

Mianyang Central Hospital, University of Electronic Science and Technology of China Gang Xie

Mianyang Central Hospital, University of Electronic Science and Technology of China

\section{Wen-yu Yang}

Chengdu University of Traditional Chinese Medicine

\section{Bin Zhang}

Mianyang Central Hospital, University of Electronic Science and Technology of China

\section{Xiao-han Li}

Affiliated Hospital of Southwest Medical University

Jun Wang

Chengdu University of Traditional Chinese Medicine

\section{Research Article}

Keywords: renal cell carcinoma (RCC), macrophage M2, extracellular vesicle (EV), miR-342-3p, NEDD4L, CEP55, E3 ubiquitin ligase, PI3K/AKT/mTOR signaling pathway, tumor growth, metastasis

Posted Date: March 3rd, 2022

DOI: https://doi.org/10.21203/rs.3.rs-1394276/v1

License: (1) (1) This work is licensed under a Creative Commons Attribution 4.0 International License. Read Full License 


\section{Abstract}

Background: Renal cell carcinoma (RCC) is an insidious and common genitourinary malignancy with the incidence rate increasing annually. Due to its difficulty in early diagnosis and lack of sensitivity to chemotherapy and radiotherapy, RCC continues being a frequent cause of cancer-related death.

Therefore, it is urgent to study the molecular mechanism of the occurrence and development of RCC and find new and reliable biomarkers or targets for its early diagnosis and treatment.

Methods: miRNA data of M2-EVs and RCC were searched on Gene Expression Omnibus (GEO) database, and potential downstream target for miRNA was predicted on a public bioinformatic database.

Transcriptional and translational levels of target genes were measured via RT-qPCR and Western blot, respectively. M2 macrophage was obtained via flow cytometry and identified by immunofluorescence staining. The morphology and particle size of M2-EVs were examined under a transmission electron microscope, and the internalization by RCC cells was monitored by fluorescence microscopy. The miR342-3p binding to NEDD4L was validated by a dual-luciferase assay. CO-IP combined with GST-pull down assay was devised to study CEP55 ubiquitination in the presence of NEDD4L. The biological abilities of RCC cells in proliferation, invasion and migration were separately tested by CCK-8 and Transwell assay. Subcutaneous tumor-bearing mouse models and lung metastasis models were built to observe in vivo role of target genes.

Results: M2-EVs induced RCC growth and metastasis. miR-342-3p showed high expression in both M2EVs and RCC cells. M2-EVs carrying miR-342-3p promoted RCC cell abilities to proliferate, invade and migrate. In RCC cells, M2-EV-derived miR-342-3p could specifically bind to NEDD4L, and consequently elevate CEP55 protein expression via suppressing NEDD4L, thereby exerting tumor-promoting effects. Further study showed that CEP55 could be degraded by ubiquitination under the function of NEDD4L, and miR-342-3p within M2-EVs facilitated the RCC occurrence and development by activating $\mathrm{PI} 3 \mathrm{~K} / \mathrm{AKT} / \mathrm{mTOR}$ signaling pathway.

Conclusion: In all, M2-EVs promote RCC growth and metastasis by delivering miR-342-3p to suppress E3 ubiquitin ligase NEDD4L and subsequently inhibit CEP55 ubiquitination and degradation via activation of the $\mathrm{PIBK} / \mathrm{AKT} / \mathrm{mTOR}$ signaling pathway, strongly driving the proliferative, migratory and invasive properties of RCC cells.

\section{Background}

Renal cell carcinoma (RCC) represents the most frequent type of renal neoplasm, accounting for $90 \%$ of adult renal malignancies and $2 \% \sim 3 \%$ of all adult malignant cancers [1-2]. It is the most lethal neoplasm of the urologic system, defined by an asymptomatic disease course, with late and uncharacteristic symptoms, leading to a poor survival prognosis [3]. Incidence of RCC has risen steadily each year since 1990s, with an approximate increase of $2 \%$ 3\% per year [2, 4]. Patients suffering from localized RCC are frequently treated with nephrectomy [5]. Unfortunately, metastasis occurs in approximate one quarter of the patients, causing extremely low survival rates and severe social burden [6-7]. Thus, it is essential to 
identify novel biomarkers for effectively monitoring RCC progression, predicting RCC prognosis, and serving as RCC therapeutic targets.

Macrophages are the most abundant immune cells in tumor microenvironment (TME), and can be categorized into the classically activated (M1) and the alternatively activated (M2) macrophages according to the polarization status [8-9]. M1 macrophages exhibit tumoricidal activities, whereas M2 macrophages facilitate tumor progression by enhancing tumor angiogenesis and metastasis [9-10]. Numerous types of cells including macrophages release extracellular vesicles (EVs), which are bioactive membrane-enclosed "packages" containing proteins, lipids and nucleic acids, that are capable of modulating the TME and affecting the signaling pathway of recipient cells [11-12]. Increasing evidences suggest that cancers highjack EV-mediated communication to facilitate tumor progression by promoting cell proliferation, sustaining angiogenesis, reprogramming energy metabolism, evading immune response, and contributing to cancer cell invasion and metastasis [12-14]. In particularly, M2 macrophage-derived EVs have been shown to stimulate the migration and invasion of various cancer types, including colorectal cancer, hepatocellular cancer and pancreatic cancer [15-17], etc. MicroRNAs (miRNAs) are one of the most studied classes of biomolecules carried by EVs. miRNAs are small noncoding RNAs that are capable of regulating gene expression post-transcriptionally, resulting in the attenuated translation of target mRNAs [18]. Several EV-derived miRNAs such as miR-19b-3p, miR-30c-5p and miR-210 have been studied and proposed as potential diagnostic and therapeutic targets in clear cell RCC (ccRCC, the most common subtype of RCC, accounting for $75 \%-80 \%$ of all RCC cases) [19-21]. However, due to the novelty of this field, the role of M2 macrophage-derived EVs (M2-EVs) in regulating RCC progression and metastasis still needs to be further elucidated.

Herein, we first determined M2-EVs-related miRNAs by bioinformatics analysis in RCC, and identified miR$342-3 p$ as the significantly up-regulated miRNAs. The role of miR-342-3p in several cancers (such as hepatocellular and nasopharyngeal carcinoma) has already been investigated [22-23]. However, the mechanism of how EV communication involving miR-342-3p affects RCC cells is not fully understood. In this study, we explored the role of M2-EV-derived miR-342-3p in RCC, and discussed the underlying mechanisms. Our findings demonstrated that miR-342-3p from M2-EVs directly targeted neural precursor cell expressed developmentally down-regulated gene 4-like (NEDD4L) and consequently regulated centrosomal protein 55 (CEP55), that contributed to PI3K/AKT/mTOR activation in RCC cells, resulting in potentiated malignant behaviors.

\section{Materials And Methods}

\section{Bioinformatics analysis}

Macrophage M2-derived EV dataset GSE97467 and RCC datasets GSE71302, GSE95384 and GSE36895 were searched from the Gene Expression Omnibus (GEO) database (https://www.ncbi.nlm.nih.gov/gds). GSE97467 contains EV data derived from THP-1 monocyte $(n=4)$ and macrophage M2 ( $n=4$ ), while GSE71302 (normal, $n=5$; tumor, $n=5$ ), GSE95384 (normal, $n=8$; tumor, 
$\mathrm{n}=8$ ) and GSE36895 (normal, $\mathrm{n}=23$; tumor, $\mathrm{n}=29$ ) includes RCC and normal renal tissue samples. Differentially expressed miRNAs which met $|\log F C|>1$ and $P$ value $<0.05$ were screened out using $R$ package "limma" package (http://www.bioconductor.org/packages/release/bioc/html/limma.html). ENCORI database (http://starbase.sysu.edu.cn/index.php) was used to obtain potential targets for miR342-3p and perform correlation analysis. Additionally, the UbiBrowser database

(http://ubibrowser.ncpsb.org/ ubibrowser/home/index) was used to predict E3 ubiquitin ligases. Starbase (http://starbase.sysu.edu.cn/) was searched to obtain binding sites of miR-342-3p and NEDD4L.

\section{Cell culture}

RCC cell lines (ACHN and 769-P), human umbilical vein endothelial cells (HUVECs) and monocyte cell line THP-1 were purchased from the Cell Bank of the Typical Culture Preservation Committee of Chinese Academy of Sciences (Shanghai, China). These cell lines were maintained in RPMI 1640 medium supplemented with $10 \%$ fetal bovine serum (FBS) at $37^{\circ} \mathrm{C}$ in a $5 \% \mathrm{CO}_{2}$ humidified incubator.

\section{Isolation of $\mathrm{CD}_{11 \mathrm{~b}}{ }^{+} / \mathrm{CD}_{163}{ }^{+} \mathrm{M} 2 \mathrm{TAMs}$}

RCC tissue samples were cut into small pieces, digested at $37^{\circ} \mathrm{C}$ and subjected to continuous rotation for removal of erythrocytes. Then, cells were incubated with Alexa Fluor 488-conjugated mouse anti-human CD11b (R\&D Systems, FAB16991G-100) and mouse anti-human CD163-APC (Miltenyi, 130-100-612) or control IgG antibodies (Miltenyi, 130-098-846 and R\&D Systems, IC0041G) at $4{ }^{\circ} \mathrm{C}$ for $30 \mathrm{~min}$.

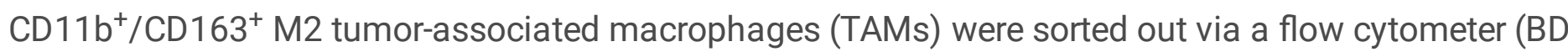
FACSAria II cell sorter).

\section{Immunofluorescent staining}

M2 TAMs were digested and cultured in immunofluorescence chamber at $2 \times 10^{5}$ cells per well. When the cell confluence reached about $90 \%$, the cells were washed with cold PBS for 3 times and fixed in $4 \%$ paraformaldehyde. After permeabilizing with $0.3 \%$ Triton $X-100$ and blocking with goat serum, the cells were incubated with the primary antibodies CD68, CD206 and CD163 (diluted in PBS at a ratio of 1:100, Abcam, Cambridge, UK) at $4{ }^{\circ} \mathrm{C}$ overnight. The primary antibodies were removed, and cells were washed and incubated with FITC-labeled fluorescent secondary antibody (sheep anti-mouse IgG H\&L) (Abcam, Cambridge, UK) at room temperature away from light for $1 \mathrm{~h}$. The cells were then exposed to DAPI (100 $\mu \mathrm{L}$ per well) for $15 \mathrm{~min}$ in dark condition. Fluorescent quench was added and the tablets were sealed and observed under fluorescence microscope (ECLIPSE E800, Nikon, Japan). The experiment was repeated three times.

\section{Lentiviral infection and plasmid transfection}


For lentiviral infection, available M2 TAMs were trypsin-digested, counted and then inoculated in 6-well plates. Lentivirus vectors containing miR-342-3p inhibitor (anti miR-342-3p) and corresponding negative control (anti NC) (Genechem Inc., Shanghai, China) (MOI=20) were transfected into M2 TAMs. Highly transfected cells were sorted out using 3-day treatment of puromycin $(1 \mu \mathrm{g} / \mathrm{mL})$. M2 TAMs were divided into the following groups: Control (normal saline), M2-CM (conditioned medium [CM] for extraction of M2 TAMs), M2-anti-NC and M2-anti miR-342-3p. TAMs of these groups were infected respectively with lentivirus for EVs isolation.

Transient transfection was fulfilled in RCC cells with pcDNA-3.1 NEDD4L, pcDNA-3.1 Smurf1, pcDNA-3.1 Smurf2, pcDNA-3.1 Itch, CEP55 shRNA, miR-342-3p mimic, miR-342-3p inhibitor and corresponding NCs (Genechemlnc., Shanghai, China) respectively. In short, RCC cells at $4 \times 10^{5}$ cells $/ \mathrm{mL}$ were counted and cultured in 6-well plates. Upon reaching $80 \%$ confluence, cell transfection was performed using Lipofectamin 2000 according to the manufacturer's manual.

\section{Isolation and identification of M2-EVs}

M2 TAMs were incubated overnight at $2 \times 10^{5}$ cells per well in 6-well plates. The culture medium was replaced with EV-free serum. After cultured for another $48 \mathrm{~h}, \mathrm{CM}$ was collected, and M2-EVs were isolated via differential centrifugation ( $500 \mathrm{~g}$ for $15 \mathrm{~min} ; 2,000 \mathrm{~g}$ for $15 \mathrm{~min} ; 10,000 \mathrm{~g}$ for $20 \mathrm{~min}$ ) at $4{ }^{\circ} \mathrm{C}$. Following filtration by a $0.22 \mu \mathrm{M}$ filter (on ice), the sample was centrifuged at $110,000 \mathrm{~g}$ for $70 \mathrm{~min}$, resuspensed using PBS (on ice) and ultracentrifuged. M2-EVs were finally obtained after resuspension in $100 \mu \mathrm{L}$ sterile PBS.

Transmission electron microscope (TEM) was adopted to validate the successful extraction of M2-EVs. Briefly, $20 \mu \mathrm{L}$ EVs were loaded onto the copper grid, followed by liquid suction with filter paper, counterstaining with the addition of $30 \mu \mathrm{L}$ phosphotungstic acid solution $(\mathrm{pH} 6.8)$, bakeing with an incandescent light and photographing. Particle size was determined by a Nanoparticle Tracking Analyzer (NS300, Malvern Instruments Ltd, UK).

Surface markers of EVs were quantitated by Western blot. In short, the concentrated EV suspension was firstly processed by a BCA kit (23227, Thermo Fisher Scientific, USA) for protein quantitation, after which SDS-PAGE was performed, and the separated proteins were transferred to a bio-membrane to determine expression of EV markers, including Alix (ab117600, 1:1000), CD63 (ab134045, 1:1000), LAMP2 (ab13524, 1:1000) and calnexin (ab22595, 1:1000). All antibodies were ordered from Abcam. Ponceau red was used as Loading Control.

\section{Fluorescent labeling and transfer of EVs}

M2-EV labeling was fulfilled with the PKH67 Green Fluorescence Kit (UR52303, Umibio (Shanghai) Co. Ltd) according to the manufacturer's instructions. Afterwards, the harvested EVs were co-cultured with 
RCC cells, fixed in 4\% paraformaldehyde, washed by PBS and exposed to 4',6-diamidino-2-phenylindole (DAPI, Sigma, D9542) for nuclear staining. The slides were photographed under the fluorescence microscope (ECLIPSE E800, Nikon, Japan).

The internalization of M2-EV-carried miR-342-3p by recipient cells (RCC cells) was further investigated. M2 macrophages were transfected with Cy3-miR-342-3p (GenePharma) using Lipofectamine 3000 (Invitrogen, Carlsbad, CA, USA) (serum-free medium) for $6 \mathrm{~h}$, and then the medium was replaced with 10\% EV-free serum medium. After further 48-h incubation, the supernatant was collected and M2-EVs carrying Cy3-miR-342-3p were extracted. The EVs were resuspended by PBS and added to RCC cells. Subsequently, the cells were fixed and washed by PBS, and the cytoskeletons were labeled with Phalloidin-iFfluor 488 Reagent (ab176753, 1:1000) for 30 min at room temperature. The nuclei were stained with DAPI. Finally, the internalization of EVs-miR-342-3p in RCC cells was validated microscopically by fluorescence microscopy (ECLIPSE E800, Nikon, Japan).

\section{Dual-luciferase reporter assay}

A dual luciferase reporter assay was done using a Promega dual-luciferase reporter assay kit (Madison, USA). The sequences of NEDD4L containing predicted miR-342-3p binding sites and mutant binding sites were ligated to the pmirGLO Dual-Luciferase miRNA Target Expression Vectors (E1330, Promega, USA) to build NEDD4L-WT and NEDD4L-MUT reporter vectors. HEK293 cells were co-transfected with the above indicated plasmids and miR-342-3p mimic or miR-NC using Lipofectamine 3000 (Invitrogen, Carlsbad, CA, USA). After $48 \mathrm{~h}$, the luciferase activities were detected. The experiment was devised with three replicates.

\section{CO-immunoprecipitation (CO-IP)}

RCC cells were collected and lysed with RIPA (C1053, Beijing Pulley Gene Technology Co., Ltd.) containing protease inhibitor cocktail (HY-K0010, MCE, Shanghai, China). The proteins obtained were incubated with primary antibodies at $4{ }^{\circ} \mathrm{C}$ overnight. Protein A/G-agarose beads (36403ES08, Yeasen, Shanghai, China) were added for $4 \mathrm{~h}$ to obtain immunoprecipitates, which were then purified for at least three times in lysis buffer. Purified complexes were treated by $2 \times$ SDS loading buffer, boiled, electrophoresed and assayed by Western blot. Antibodies used include: anti-Flag (AE005, 1:100, mouse antibody, abclonal), and anti-Myc (AE070, 1:50, rabbit antibody, abclonal).

\section{GST Pull-down assay}

E. coli (BL21 DE3 strain) transformed with GST-NEDD4L or His-CPE55 vectors was induced with $0.4 \mathrm{mM}$ IPTG at $16^{\circ} \mathrm{C}$ for $16 \mathrm{~h}$, to express GST-NEDD4L or His-CPE55 proteins. Purified GST-NEDD4L proteins (200 ng) were immobilized on glutathione-agarose beads (G0924-1 ML, Millipore, USA), which were then incubated with purified HIS-CPE55 proteins (200 ng). GST protein alone served as a negative control. 
After washing, Western blot assay was performed to analyze the proteins bound to the beads using antiHis (catalog sc-803, Santa Cruz Biotechnology Inc., 1:1000) or anti-GST antibody (catalog sc-138, Santa Cruz Biotechnology Inc., 1:5000).

\section{Endogenous ubiquitination analysis}

HA-Ub, Myc-NEDD4L, Myc-NEDD4L-MUT or Flag-CEP55 plasmids were transfected into RCC cells, followed by $4 \mathrm{~h}$ treatment with $10 \mu \mathrm{M}$ MG132 (MedChemExpress, Shanghai, China). Afterwards, cell lysis was performed using $100 \mu \mathrm{L}$ conventional lysis buffer. The products were denatured $\left(95^{\circ} \mathrm{C}, 5 \mathrm{~min}\right)$, added with 1\% SDS and incubated in anti-Flag antibody (AE005, 1:100, mouse antibody, abclonal) and protein G-agarose (11243233001, Roche, Shanghai, China) successively. Anti-ubiquitin antibody was used to determine the endogenous ubiquitination of CEP55 in immunoprecipitates by Western blot.

\section{Protein half-life assay}

RCC cells were treated with $10 \mu \mathrm{M}$ cycloheximide (CHX, MedChemExpress, Shanghai, China) for various periods of time $(0,0.5,1$ and $2 \mathrm{~h})$, or treated with MG132 to block protein synthesis at the same time. Cell extracts were prepared, and protein expressions were measured by Western blot assay.

\section{CCK-8 assay}

The 96-well culture plates were used to culture the cells. According to the manufacturer's instructions, 10 $\mu \mathrm{L}$ CCK-8 (Sigma, USA) was added to each well, and after being incubated in the cell incubator for $1 \mathrm{~h}$, the absorbance at $450 \mathrm{~nm}$ was measured on the enzyme labeling instrument (NYW-96M, Beijing Nuoyawei instrument Co., Ltd.). The cell viability curve was drawn with time point as abscissa and OD value as ordinate. The experiment was repeated three times.

\section{Transwell assay}

Cells were added to the upper Transwell chambers (24-well insert; pore size, $8 \mu \mathrm{m}$; Corning, USA) precoated without (migration assay) or with (invasion assay) $50 \mu \mathrm{L}$ of diluted Matrigel (1:8 dilution; BD Biosciences). Medium containing 10\% FBS was added to the lower Transwell chambers. After $48 \mathrm{~h}$, the transmigrated cells were fixed with $4 \%$ paraformaldehyde, treated with $0.2 \%$ Triton X-100 (Sigma, USA) solution and stained with $0.05 \%$ gentian purple. The number of stained cells was counted under inverted microscope (XDS-800D, Shanghai Caikang Optical Instrument Co., Ltd., Shanghai, China) to evaluate the cell migration and invasion capabilities. Five visual fields were randomly selected to count, and the number of cells was expressed by mean. 


\section{RT-qPCR}

Total RNA extracted from tissues or cells using Trizol (Thermo Fisher Scientific, New York, USA) was taken to perform reverse transcription to obtain CDNA or miRNA CDNA with a reverse transcription kit (Fermentas Inc., Ontario, CA, USA) or miRcute Plus miRNA First-Strand cDNA Synthesis Kit (TIANGEN, China). Synthetic exogenous internal reference cel-miR-39 (1 pmol per sample; TIANGEN, China) was supplemented to culture medium $(350 \mu \mathrm{L})$ or EVs $(100 \mu \mathrm{g})$ prior to the experiment. Subsequently, miRNA was isolated from these samples using the mirVana PARIS kit (Ambion, USA). mRNA RT-qPCR was completed with SYBR ${ }^{\circledR}$ Premix Ex Taq (Takara, Japan) on the ABI StepOne real-time PCR system (Applied Biosystems, USA). Meanwhile, miRcute Plus miRNA qPCR detection kit (TIANGEN, China) was adopted in miRNA RT-qPCR. GAPDH was used as the internal control in measuring mRNA expression in cells and tissues. U6 was used as the internal control in measuring miRNA expression. In addition, miRNA level was normalized relative to exogenouscel-miR-39. The miRNA universal reverse primer was obtained from miRcute Plus miRNA qPCR detection kit (TIANGEN, China), and other primers were provided by Shanghai Sangon (see Table 1 for primer sequences). All experiments were processed in triplicates and the results were analyzed following the $2^{(-\Delta \Delta C T)}$ method.

Table 1

RT-qPCR primer sequence

\begin{tabular}{|c|c|}
\hline Gene & Primer sequence $₫ 5^{\prime}-3^{\prime} \rrbracket$ \\
\hline \multirow[t]{2}{*}{ miR-342-3p (human) } & F: TCTCACACAGAAATCGC \\
\hline & $\mathrm{R}$ : universal primer \\
\hline \multirow[t]{2}{*}{ cel-miR-39 (human) } & F: GGTCACCGGGTGTAAATCAGCTTG \\
\hline & R: universal primer \\
\hline U6 snRNA (human) & F: CTCGCTTCGGCAGCACA \\
\hline \multirow[t]{2}{*}{ NEDD4L (human) } & F: GACATGGAGCATGGATGGGAA \\
\hline & R: GTTCGGCCTAAATTGTCCACT \\
\hline \multirow[t]{2}{*}{ GAPDH (human) } & F: CATCTTCTTTTGCGTCGCCA \\
\hline & R: TTAAAAGCAGCCCTGGTGACC \\
\hline
\end{tabular}

\section{Western blot}

Total protein was extracted from tissues or cells using RIPA lysate containing PMSF (Beijing Applygen). Protein concentration was determined based on the instructions of a bicinchoninic acid (BCA) protein 
assay kit (Thermo Fisher Scientific, USA). The protein bands were separated by SDS-PAGE electrophoresis and then transferred to PVDF membrane (Millipore, USA). The membrane was blocked with $5 \%$ skim milk for $1 \mathrm{~h}$. Primary antibodies (all from Abcam) against NEDD4L (ab46521, 1:1000), CEP55 (ab170414, 1:1000), PI3K (ab16502, 1:500), AKT (ab8805, 1:1000), p-AKT (ab38449, 1:1000), mTOR (ab32028, 1:1000), p-mTOR (ab109268, 1:1000), E-cadherin (ab15148, 1:500), Vimentin (ab91547, 1:1000) and GAPDH (ab8245, 1:500) were added for incubation at $4{ }^{\circ} \mathrm{C}$ overnight. The membranes were washed for three times with PBST, and HRP-coupled goat anti-rabbit IgG second antibody (ab150077, 1:1000, Abcam) was added and incubated for $1 \mathrm{~h}$ at room temperature. PBST washing was then performed. ECL solution (Biomiga, USA) combined with a Bio-Rad Gel Doc EZ Imager (Bio-Rad, Hercules, CA, USA) was applied for images capture, and Image Pro Plus 6.0 software (Media Cybernetics, USA) was used for quantitation. The density of each protein band was normalized to GAPDH. Semi-quantitative analysis was performed based on the ration of the mean gray value of target protein to the mean gray value of internal reference.

\section{Xenograft tumor and lung metastasis in nude mice}

Forty 4-5-week immunodeficient nude mice (Bal B/c, nu/nu) were housed under non-pathogenic conditions at $20-26^{\circ} \mathrm{C}$ and humidity $50-65 \%$. ACHN RCC cells or ACHN cell lines stalely transfected with pcDNA-3.1-NEDD4L were collected, counted, and resuspended in PBS with the final concentration of $2 \times 10^{7}$ cells $/ \mathrm{mL}$. In addition, trypan blue exclusion determined that $95 \%$ of the cells were viable prior to injection. The prepared ACHN RCC cells were subcutaneously injected into the back of nude mice. Eight days after injection, Dil-labeled EVs or blank control PBS was injected through the tail vein. After that, the tumor volume was measured at six-day intervals with a vernier caliper and calculated as $W=1 / 2 * a * b^{2}$ (a, long diameter; $b$, short diameter). Four weeks later, $\mathrm{CO}_{2}$ was used to execute nude mice and tumor tissues was isolated and weighed. The nude mice were randomly assigned to: i. Control (tail vein injection of saline); ii. M2-EVs (tail vein injection of $10 \mu \mathrm{g} \mathrm{M2-EVs);} \mathrm{iii.} \mathrm{M2-EVs-anti} \mathrm{miR-342-3p} \mathrm{(tail} \mathrm{vein} \mathrm{injection} \mathrm{of}$ $10 \mu \mathrm{g}$ EVs isolated from M2 macrophages infected with $10 \mu \mathrm{g}$ anti-miR-342-3p lentivirus); iv. Overexpressed (oe)-NEDD4L (injection of stable pcDNA-3.1-NEDD4L transduced ACHN cell line and tail vein injection of saline); v. oe-NEDD4L+M2-EVs (injection of stable pcDNA-3.1-NEDD4L transduced ACHN cell line and tail vein injection of M2-EVs).

To establish lung metastasis model, ACHN RCC cells or ACHN cells stably transfected with pcDNA-3.1NEDD4L were injected into female Bal B/C nude mice through the tail vein $\left(1 \times 10^{6}\right.$ cells $\left./ 100 \mu \mathrm{L}\right)$. After 14 days, $10 \mu \mathrm{g}$ Dil-labeled EVs or equal amount of PBS was repeatedly injected into nude mice via a caudal vein twice a week for a total of one month. Subsequently, all nude mice were euthanized. Then, metastatic pulmonary nodes were counted and the lung weight was measured. Lung tissues were harvested to evaluate the expressions of miR-342-3p, NEDD4L and CEP55 via RT-qPCR and Western blot. The lung was split for further H\&E staining. 


\section{Statistics}

All data were processed by SPSS 21.0 statistical software (SPSS, Inc., Chicago, IL, USA) and GraphPad Prism 8.0.2 software (GraphPad Software, Inc., La Jolla, CA, USA). Measurement data were expressed in the form of Mean \pm standard deviation (SD). Data of the two groups were compared using independent sample T test. One-way ANOVA (followed by Tukey post-hoc test) was electively used for data comparison between multiple groups. Data were compared between groups at different time points using repeated measures ANOVA and Tukey post-hoc test. Values of $P<0.05$ were considered statistically significant.

\section{Results}

\section{M2-EVs promote biological behaviors of RCC cells}

M2-EVs are documented to be a player in cell migration and metastasis in several types of cancers[24]. To investigate the pivotal biological effects of M2-EVs on RCC, M2 TAMs were firstly extracted (Figure 1-A), and presented with positive expressions of markers CD68, CD163 and CD206 in immunofluorescence staining (Figure 1-B). M2-EVs were subsequently extracted. TEM manifestations include the round or oval M2-EVs, which were uneven in size with a complete membrane structure, and contained low-density substances (Figure 1-C). Particle diameter of the EVs was 30-100 nm (Figure 1D). Western blot assay obtained positive Alix, CD63 and LAMP2 while negative calnexin proteins in EVs (Figure 1-E). PKH67 labeled M2-EVS was co-cultured with RCC cells. PKH67 labeled green fluorescence could be observed in RCC cells (Figure 1-F), indicative of the internalization of M2-EVs by RCC cells (Figure1-F). The biological effect of M2-EVs was further analyzed. Compared to the control group, M2-CM and M2-EVs groups showed a significant increase in cell proliferation (Figure 1-G), invasion (Figure 1-H)and migration (Figure 1-I). Besides, E-cadherin levels were distinctly down-regulated, while Vimentin levels were up-regulated significantly (Figure 1-J) (all $P<0.001$, Annex I). These results identify M2-EVs can promote RCC cell abilities to proliferate, migrate and invade.

\section{M2-EVs derived miR-342-3p facilitates biological behaviors of RCC cells}

M2-EVs have been shown to facilitate the motility of cancer cells. To know the underlying mechanism of action, M2-EVs enriched miRNA data in GSE97467 and differentially up-regulated miRNA data in GSE71302 and GSE95384 were selected and intersected with miR-342-3p identified, which displayed high expression in both M2-EVs and RCC samples (Figure 2-A-D). Expression of miR-342-3p in M2-EVs, HUVECEVs and monocyte THP-1-derived EVs was measured via RT-qPCR. The results showed that there was significant difference in the expression of miR-342-3p in the observed cell-derived EVs $(F=21.302, P]$ 0.001). Compared with other cell-derived EVs, miR-342-3p expressed highest in M2-EVs, but its increase was not statistically different from THP-1-EVs ( $q=2.169, \mathrm{P}=0.463)$ (Figure 2-E). RCC cells were co-cultured 
with TAMs containing Cy3-miR-342-3p. Microscopically red fluorescence was visible in RCC cells (Figure 2-F). Besides, miR-342-3p level was found to elevate in both M2-CM (ACHN: q=7.874, $P=0.003$; 769-P: $\mathrm{q}=6.641, P=0.008)$ and M2-EVs (ACHN: $\mathrm{q}=12.326, P<0.001 ; 769-\mathrm{P}: \mathrm{q}=8.169, P=0.003)$ groups when compared with control group, and the elevated level inM2-EVs group was higher than that in M2-CM group (all $P<0.05$, Annex I) (Figure 2-G). These results suggest that M2 TAMs can deliver miR-342-3p to RCC cells via EVs.

To know more about the role of miR-342-3p in M2-EVs, miR-342-3p expression was interfered in M2 TAMs. The results showed that the miR-342-3p level of M2-EVs in anti-miR-342-3p group was significantly lower than that in anti-NC group. ( $\mathrm{t}=11.244, P \otimes 0.001)$ (Figure 3-A). Subsequently, M2-EV-anti miR-342-3p was co-cultured with RCC cells, and miR-342-3p expression in RCC cells was tested to show a significant difference when compared with the control group ( $A C H N: F=29.018, P<0.001 ; 769-P: F=37.155$, $\mathrm{P}<0.001)$. Compared with M2-EV-anti NC group, the expression of miR-342-3p in M2 EVs anti miR-342-3p group decreased more significantly ( $A C H N$ : $q=7.864, P=0.004$; 769-P: $q=11.350, P=0.001$ ) (Figure 3B). Further biological experiments showed significantly elevated cell viability (ACHN: $F=16.220, P<0.001$; 769-P: $F=19.721, P<0.001$. Figure 3-C), invasion (ACHN: $F=64.249, P<0.001 ; 769-P: F=75.923$, $P<0.001$. Figure $3-D$ ) and migration ( $A C H N: F=73.947, P<0.001 ; 769-P: F=56.465, P<0.001$. Figure $3-E$ ) in the presence of M2-EVs-anti NC as compared to the control, while reverse trends were observed in M2EVs-anti miR-342-3p group versus M2-EVs-anti NC group (all $P<0.001$, Annex I) (Figure 3-C-E). In the further Western blot assay for epithelial-mesenchymal transition (EMT)-related proteins in RCC, there was a significant decline in E-cadherin ( $A C H N$ : $q=16.459, P<0.001$; 769-P: $q=14.352, P<0.001$ ) while an evident elevation in Vimentin (ACHN: $q=17.045, P<0.001 ; 769-P: q=13.716, P<0.001$ ) in M2-EVs-anti NC group versus control. To the contrary, opposite expressing trend was noted in M2-EVs-anti miR-342-3p group versus M2-EVs-anti NC group (E-cadherin, $A C H N$ : $q=14.846, P<0.001 ; 769-P: q=13.360$, $P<0.001$; Vimentin, $q=15.534, P<0.001 ; 769-P: q=12.640, P<0.001$ ) (Figure 3-F). Altogether, M2-EVs derived miR-342-3p can promote RCC cell viability and motility.

\section{M2-EVs-miR-342-3p prevents CEP55 degradation by targeted inhibition of NEDD4L in RCC cells}

It was proven that CEP55 shows adundant expression and plays a tumor-promoting role in RCC[5]. $\mathrm{H}$ Chen et al. also found that CEP55 potentiated EMT of RCC cells via activating PI3K/AKT/mTOR pathway[25]. Additionally, the ENCORI data implied a positive correlation of miR-342-3p with CEP55 in RCC (Figure 4-A).

To further investigate whether CEP55 is involved in M2-EVs-miR-342-3p dependent regulation in the tumorigenesis and development of RCC, potential downstream target genes for miR-342-3p were obtained on ENCORI and were then intersected with the differentially down-regulated genes in GSE36895 dataset and the E3 ubiquitin ligase of CEP55 predicted by the UbiBrowser database (Figure 4-B-C). In the 
meantime, ENCORI data revealed a negative correlation between NEDD4L and CEP55 in RCC (Figure 4D). Therefore, NEDD4L was eventually selected as the target gene.

A retrieval on Starbase showed potential miR-342-3p binding sites on NEDD4L 3'UTR (Figure 4-E). The dual-luciferase assay indicated a decline in luciferase signal in NEDD4L-WT group versus mimic NC group ( $\mathrm{t}=13.273, P<0.001)$, yet the signal varied not statistically in NEDD4L-MUT group versus mimic NC group ( $\mathrm{t}=0.170, P=0.873$ ) (Figure $4-\mathrm{F})$. Thus, miR-342-3p can specifically inhibit the NEDD4L gene.

The associations among miR-342-3p, NEDD4L and CEP55 were further investigated. We then transfected RCC cells with miR-342-3pmimic/inhibitor. As detected by Western blot, no expression difference was observed in mimic-NC group versus inhibitor-NC group (all $P>0.001$, Annex I). In miR-342-3p mimic group versus mimic-NC group, miR-342-3p and CEP55 expressions were remarkably increased and NEDD4L expression was observably decreased (all $P<0.001$, Annex I). In miR-342-3p inhibitor group versus inhibitor-NC group, miR-342-3p, CEP55 and NEDD4L showed the reverse expression trend (all $P<0.001$, Annex I) (Figure 4-G). Furthermore, RCC cells were treated with M2-EVs. It was observed that NEDD4L expression was significantly decreased (ACHM: $F=76.823, P<0.001 ; 769-P$ : $F=70.154, P<0.001$ ) while CEP55 expression was increased (ACHM: $F=78.031, P<0.001 ; 769-\mathrm{P}: \mathrm{F}=41.620, P<0.001$ ) compared with the control group. To the contrary, NEDD4L (ACHM: $q=14.487, P<0.001 ; 769-\mathrm{P}: \mathrm{q}=13.379, P<0.001$ ) showed elevated expression and CEP55 (ACHM: $\mathrm{q}=14.842, P<0.001 ; 769-\mathrm{P}: \mathrm{q}=10.641, P<0.001$ ) displayed reduced expression in M2-EVs-anti miR-342-3p group versus M2-EVs-anti NC group (Figure 4-

$H)$. Collectively, the data indicate that M2-EVs-miR-342-3p can prevent CEP55 degradation by suppressing NEDD4L in RCC cells.

\section{M2-EVs-miR-342-3p/NEDD4L/CEP55 axis enhances RCC growth and metastasis}

To further investigate the involvement of NEDD4L/CEP55 axis in miR-342-3p dependent mechanism of action in RCC, we transfected RCC cells with pcDNA-3.1-NEDD4L (oe-NEDD4L) and extracted M2-EVS to treat RCC cells. In Western blot, NEDD4Lexpression (ACHN: $q=20.427, P \otimes 0.001 ; 769-P: q=13.999, P \otimes 0.001)$ was increased and CEP55 expression (ACHN: $q=17.728, P \otimes 0.001 ; 769-P: q=15.175, P \otimes 0.001)$ was significantly decreased in oe-NEDD4L group versus oe-NC group. Meanwhile, NEDD4L (ACHN: q=18.172, $P \otimes 0.001$; 769-P: $q=13.294, P \otimes 0.001$ ) was starkly downregulated and CEP55 (ACHN: $q=15.814, P \otimes 0.001$; 769-P: q=13.404, $P \otimes 0.001$ ) was sharply upregulated in oe-NEDD4L+M2-EVs group versus oe-NEDD4L group (Figure 5-A).

Subsequently, biological functional experiments were devised to uncover significantly weakened cell viability (all $P<0.001$, Annex I) (Figure 5-B), migration (ACHN: q=16.036, $P \otimes 0.001 ; 769-\mathrm{P}$ : $\mathrm{q}=13.803, P]$ 0.001 ) (Figure $5-C$ ) and invasion ( $A C H N: q=14.363, P \otimes 0.001 ; 769-P: q=10.106, P \bowtie 0.001$ ) (Figure 5-D) in oeNEDD4L group versus oe-NC group, while profoundly enhanced abilities of RCC cells after M2EVs application when compared with oe-NEDD4L alone (all $P<0.001$, Annex I) (Figure 5-B-D). As regards 
EMT markers, NEDD4L overexpression led to increased E-cadherin while decreased Vimentin compared with oe-NC group, which could be restored after M2-EVs treatment (all $P<0.001$, Annex I) (Figure 5-E).

In vivo animal experiment was then conducted. Empty RCC cells or cells overexpressing NEDD4L were respectively subcutaneously injected into the nude mice, along with tail injection of M2-EVs. It was found that NEDD4L overexpression resulted in reduced tumor growth speed and weight, while the presence of M2-EVs exerted a tumor-promoting effect. Additionally, the tumor growth speed and weight tended to be significantly reduced in oe-NEDD4L+M2-EVs group and M2-EVs-anti miR-342-3p group versus M2-EVs group (all $P<0.001$, Annex I) (Figure 6-A-C).

In lung metastasis models, lung metastasis was much frequent in control ( $q=11.006, P \otimes 0.001)$ and M2EVs ( $q=43.644, P \otimes 0.001)$ groups versus oe-NEDD4L group. In the meantime, the metastatic potential in M2-EVs group was also much higher as compared to oe-NEDD4L+M2-EVs group ( $q=31.120, P \otimes 0.001)$ and M2-EVs-anti miR-342-3p group (q=31.120, P冈0.001) (Figure 6-D-F). Dillabeled EVs could be observed in the metastatic lung tissue (Figure 6-G). Furthermore, miR-342-3p, NEDD4L and CEP55 levels were measured in lung tissue of each group. As analyzed, relative to control group, in M2-EVs group, miR-342$3 p(q=16.455, P \otimes 0.001)$ (Figure 6-H) and CEP55 ( $q=20.732, P \otimes 0.001)$ (Figure 6-I) levels were elevated and NEDD4L ( $q=30.413, P \otimes 0.001)$ (Figure 6-I) was reduced. NEDD4L (q=16.870, P冈0.001) (Figure 6-I) showed higher expression while CEP55 ( $q=27.814, P \otimes 0.001)$ (Figure 6-I) was lower expressed in oe-NEDD4L versus control. In comparison to M2-EVs, the increase of miR-342-3p (q=10.761, Pख0.001) (Figure 6-H) and CEP55 ( $q=17.514, P \otimes 0.001$ ) (Figure 6-I) levels in oe-NEDD4L+M2-EVs group was much more significant. Comparing M2-EVs group to M2-EVs-anti miR-342-3p group, a profound increase in miR-342-3p $(\mathrm{q}=18.352, P \otimes 0.001)$ (Figure 6-H) and CEP55 ( $\mathrm{q}=16.222, P \otimes 0.001)$ (Figure 6-I) expressions and a reduction in NEDD4L (q=30.887, $P \otimes 0.001$ ) (Figure 6-I) were noted (all $P<0.05$ ). Taken together, M2-EVs-miR-342-3p promotes growth and metastasis of RCC via the NEDD4L/CEP55 axis.

\section{M2-EVs-miR-342-3p mediates E3 ubiquitin ligase NEDD4L to affect CEP55 degradation}

Existing research demonstrated that NEDD4L as an E3 ubiquitin ligase can promote the degradation of related proteins. Besides, CEP55 was reported to promote the EMT of RCC cells via activating PI3K/AKT/mTOR pathway. It was thus speculated that M2-EVs-miR-342-3p participates in RCC occurrence and progression probably via the NEDD4L/CEP55/PI3K/AKT/mTOR axis.

To assess whether CEP55 can be ubiquitinated and degraded by the E3 ubiquitin ligase NEDD4L in RCC, CEP55 protein expression was measured in RCC cells. It was revealed that CEP55 protein decreased with prolonged time in the presence of $\mathrm{CHX}$, as compared to dimethyl sulfoxide(DMSO) group (all $P<0.001$, Annex I), while the decrease slowed after CHX-MG132 treatment (all $P<0.001$, Annex I) (Figure 7A). Following overexpression treatment for ubiquitin ligases Smurf1, Smurf2, Itch and NEDD4L, decline of CEP55 was only observed upon NEDD4L overexpression (all $P<0.001$, Annex I, oe-NEDD4L group 
compared with other groups) (Figure 7-B), which was suppressed after MG132 intervention ( $\mathrm{q}=15.469, P<0.001$; oe-NEDD4L+DMSO vs. oe-NEDD4L+MG132) (Figure 7-C).

CO-IP and GST pull down assays were performed in the presence of MG132 to test the relationship between NEDD4L and CEP55 at the protein level. In the meantime, endogenous CEP55 was detected after expression of Myc-labeled NEDD4L in cells. As displayed in Figure 7-D, Myc-NEDD4L could be coprecipitated with anti-CEP55 antibodybut not with control immunoglobulin IgG. It reveals the interaction of NEDD4L with endogenous CEP55 in RCC cells.

To validate the direct interaction between the two, GST-pulldown assay was conducted with in vitro synthetic His-CEP55 and GST-NEDD4L (Figure 7-E). The effect of NEDD4L overexpression on CEP55 ubiquitination was then explored. As shown in Figure 7-F, NEDD4L could promote the ubiquitination and degradation of CEP55, while such effect was reduced when mutations occurred in NEDD4L. This implies that CEP55 ubiquitination and degradation can be modulated by E3 ubiquitin ligase NEDD4L.

Finally, to gain more insight into the miR-342-3p/NEDD4L/CEP55/PI3K/AKT/mTOR axis, CEP55 was silenced in RCC cells followed by co-culture with M2-EVs. In the results, CEP55 expression (ACHN: $q=16.059, P \otimes 0.001 ; 769-P: q=15.900, P \otimes 0.001)$ was silenced after short hairpin(sh)CEP55 transfection, along with reduction in PI3K (ACHN: q=15.259, P凶0.001; 769-P: q=1476, $P$ ] 0.001 ) expression and decrease in phosphorylation levels of AKT-Ser473 (ACHN: $q=15.007, P \otimes 0.001 ; 769-$ $P: q=8.861, P \otimes 0.001)$ and mTOR-ser2448 (ACHN: $q=13.166, P \otimes 0.001 ; 769-P: q=9.915, P \otimes 0.001$ ), yet NEDD4L expression was not significantly changed (ACHN: $q=0.746, P=0.861 ; 769-P: q=0.792$, $P=0.846)$. Nevertheless, compared with sh-CEP55, co-culture with sh-CEP55 and M2-EVs decreased NEDD4L (ACHN: $q=15.010, P \otimes 0.001 ; 769-P: q=13.879, P \otimes 0.001)$ protein expression and posed promotive effects on CEP55 (ACHN: $q=14.662, P \otimes 0.001 ; 769-P: q=14.582, P \otimes 0.001)$ and PI3K (ACHN: $q=13.293, P]$ $0.001 ; 769-\mathrm{P}: \mathrm{q}=15.676, P \otimes 0.001)$ protein levels, as well as phosphorylation levels of AKTS473 (ACHN: q=12.536, $P \otimes 0.001 ; 769-\mathrm{P}: \mathrm{q}=9.761, P \otimes 0.001$ ) and mTOR-ser2448 (ACHN: $\mathrm{q}=11.645, P \otimes 0.001$; 769-P: q=8.601, $P \otimes 0.001$ ) were increased (Figure 7-G).

Altogether indicates that M2-EVs inhibit the E3 ubiquitin ligase NEDD4L to prevent the ubiquitination and degradation of CEP55 and activate the PI3K/AKT/mTOR signaling pathway.

\section{Discussion}

It is well established that the formation and maintenance of a cancer niche deeply depend on their surrounding microenvironment that consists of a network of reciprocal cell types such as endothelial cells, inflammatory cells, fibroblasts, stem cells, and immune cells $[12,27]$. TAMs are the most prominent tumor-infiltrating immune cells within the TME and despite growing research, the regulatory crosstalk between TAM and RCC cells, especially how macrophages modulate various hallmarks of RCC during tumor progression remains not completely understood. Here, we found that elevated exogenous miR-342$3 p$ within M2-EVs effectively disrupted NEDD4L expression and thus prevented the ubiquitination and 
degradation of CEP55. The upregulated CEP55 subsequently activated the PI3K/AKT/mTOR pathway, and strongly induced the proliferative, migratory and invasive properties of RCC cells.

Desregulated miRNAs play a critical role in carcinogenesis [28]. miR-342-3p, localized to $14 q 32$, has emerged as an important cancer-related miRNA in human cancers [29]. This miRNA is frequently downregulated in hepatocellular carcinoma, non-small cell lung cancer, and gallbladder cancer, and acts as a tumor suppressor [22, 30-31]. However, its functional significance in RCC is still poorly elucidated. In this study, we intriguingly verified a significant up-regulated expression of miR-342-3p in RCC tissues compared with normal controls, revealing that miR-342-3p was a carcinogenic factor rather than a suppressor implicated in development and progression of RCC. In addition, we uncovered the highly expressed miR-342-3p in M2 macrophage-derived EVs. Accumulating evidence has supported the important roles of EV-derived miRNAs in RCC [20-21, 32]. For instance, serum exosomal miR-210 originating from tumor tissue has been regarded as a novel diagnostic marker and prognostic predictor for ccRCC progression [21]. Additionally, urinary exosomal miR-30c-5p targets heat-shock protein 5 (HSPA5) and inhibits ccRCC progression, that has considerable potential as a diagnostic biomarker for early-stage ccRCC [20]. Moreover, EVs shuttled miR-31-5p can transfer resistance information and promote sorafenib resistance in RCC by directly targeting MutL homolog 1 (MLH1), thereby both miR-31$5 p$ and its target gene are likely to be predictive biomarkers and therapeutic targets for sorafenib resistance [32]. In current work, we demonstrated that M2-EVs could carry and transmit miR-342-3p into RCC cells, thereafter to drive RCC proliferative, migratory and invasive capacities in vitro and in vivo. This observation indicated the miR-342-3p appeared to be a novel and effective therapeutic target gene for RCC.I

Another key important observation in this study was that miR-342-3p could bind to NEDD4L and downregulate its expression. NEDD4L is an E3 ubiquitin ligase that regulates channel internalization and turnover [33]. It appears to possess roles in multiple cell processes, such as transporter modulation, autophagy and signal transduction [33]. NEDD4L level is significantly changed, and it exhibits distinct functions in different carcinomas by regulating of certain major pathways (such as TGF- $\beta$, WNT and EGFR signaling pathways) [34-35]. In gallbladder cancer, NEDD4L is significantly up-regulated and exerted pro-oncogenic role through regulation of matrix metallopeptidase 1 and 13 genes transcription [36], whereas in prostate cancer, down-regulation or loss of function of NEDD4L (that can exert an antitumor activityvia regulating the TGF $\beta 1$ signaling) is observed and proposed to be associated with the malignancy [37]. Also, decreased NEDD4L expression in NSCLC is found to be more tumor aggressive, and could predict poorer survival time $[34,38]$. Here, we showed that NEDD4L expression was decreased in RCC cell lines by miR-342-3p delivered in M2-EVs, thus consequently promoting the RCC cell migration and invasion. Our observation was inconsistent with other reports of the low expression of NEDD4L in ccRCC $[37,39]$. All these elucidated the tumor suppressive role of NEDD4 in RCC carcinogenesis. Meanwhile, we found that transfer of M2-EVs-drived miR-342-3p into RCC cells to target NEDD4L diminished the degradation of CEP55. NEDD4L has been widely reported to modulate multiple signaling pathways in tumor cells through the ubiquitination and degradation pathway [40-41]. For example, ERBB3 (HER3), one of the EGFR family proteins, can undergo NEDD4L-mediated ubiquitination and 
degradation to down-regulate the signal transduction pathways of occurrence and development in cancers [42]. Additionally, NEDD4L is involved in uncoordinated 51-like kinase 1 (ULK1) ubiquitination and degradation, thereby playing vital roles in initiating autophagy and maintaining redox homeostasis [43-44]. Our study had verified the negative regulatory relationship between NEDD4L and CEP55, thus the above reports would support our hypothesis that NEDD4L exerts the ubiquitination regulation effect on CEP55. CEP55 is a centrosome- and midbody-associated protein that is critical for cell cycle progression and cytokinesis [45]. Many studies have revealed that CEP55 serves pivotal roles in the cell cycle and survival through regulation of the PI3K/AKT pathway [45-47]. For instance, Li et al. has showed that CEP55 can promote proliferation and inhibit apoptosis via PI3K/AKT/p21 signal pathways, further attributing to the carcinogenesis and progression of glioma cells [46]. Additionally, Chen et al. has demonstrated that PI3K/AKT/mTOR pathway is capable of regulating the effects of CEP55 on the migration, invasion and EMT of RCC cells and might be used as an effective prognostic marker [47]. In consistent with this, our study also verified that up-regulated CEP55 could sustain RCC growth, proliferation and metabolism through activation of the PI3K/AKT/mTOR pathway. In conjunction with existing evidence, we preliminarily indicated that M2-EVs encapsulated miR-342-3p could promote the CEP55 expression by targeting NEDD4L and inhibiting NEDD4L expression, thus consequently promoting the proliferative, migratory and invading properties of RCC cells via activation of the PI3K/AKT/mTOR pathway.

In fact, PI3K/AKT pathway is modestly mutated but highly activated in RCC [48]. Recent studies have evidenced that classical activation of PI3K/AKT network is not always triggered by extracellular stimuli and transmembrane protein receptors [48]. Currently, miRNAs are emerging as a new class of important regulators of the PI3K/AKT pathway. For example, miR-122 is proved to be a positive modulator of $\mathrm{PI3K} / \mathrm{AKT}$ signaling, that can promote proliferation, invasion, and migration of RCC cells [49]. Additionally, miR-182-5p is in a down-regulated expression pattern of AKT, and its reduction results in AKT activation and subsequent RCC proliferation [50]. These results partially supported that miR-342-3p could be identified to be a novel regulator that contributed to aberrant PI3K/AKT pathway activation in RCC, representing a promising target for RCC therapy. Moreover, some previous study has reported that in the presence of the tumors, EV-enrichment may represent an epigenetic silencing mechanism whereby ccRCC maintains tumor development and growth by activating the PI3K/AKT pathway [32]. The overall activation of PI3K/AKT in CCRCC is higher than in other cancers, suggesting that dysregulation of the $\mathrm{PI} 3 \mathrm{~K} / \mathrm{AKT}$ pathway in RCC may be a consequence of EV-mediated epigenetic mechanisms $[48,51]$.

Altogether, findings obtained in our study concluded that M2-EVs-drived miR-342-3p could play an important role in the invasion and migration process of RCC, and had the potential to be used as therapeutic biomarker. However, we recognize that the additional mechanisms may be involved in such pathways, which warrants further exploration.

\section{Conclusion}


To sum up, a preliminary conclusion can be obtained in this research that, $\mathrm{M} 2$ macrophages may carry miR-342-3p through EVs to target the E3 ubiquitin ligase NEDD4L in RCC cells, inhibit the ubiquitination degradation of CEP55 and activate the PI3K/AKT/mTOR signaling pathway, thereby promoting the growth and metastasis of RCC (Fig. 8). This finding provides a novel therapeutic target in future RCC treatment.

\section{Abbreviations}

CEP55: centrosomal protein 55; CO-IP: CO-immunoprecipitation; EMT: epithelial-mesenchymal transition; EVs: extracellular vesicles; FBS: fetal bovine serum; GEO: Gene Expression Omnibus; HSPA5: heat-shock protein 5; HUVECs: human umbilical vein endothelial cells; NEDD4L: neural precursor cell expressed developmentally down-regulated gene 4-like; NHC, National health commission; RCC: Renal cell carcinoma; TAMs: tumor-associated macrophages; TEM: Transmission electron microscope; TME: tumor microenvironment

\section{Declarations}

\section{Ethics approval and consent to participate}

All research procedures were conducted with approval of the Ethics Committee of Mianyang Central Hospital and in line with the Declaration of Helsinki. All patients and/or legal guardians signed the informed consent documentation prior to experiments. Additionally, all animal experiments were approved by the Animal Ethics Committee of Mianyang Central Hospital. Great efforts were made to minimize the number of animals used in the experiments and their suffering.

\section{Consent for publication}

Not applicable.

\section{Availability of data and materials}

The datasets used and/or analysed during the current study are available from the corresponding author on reasonable request.

\section{Competing interests}

All authors declare no financial competing interests.

All authors declare no non-financial competing interests. 


\section{Funding}

This work was supported by the Science and Technology Department of Sichuan Province (2015SZ0117, 2019YJ0701, and 2021YJ0239).

\section{Authors' contributions}

JFF, BX and CMD designed and conceived the study, and were the major contributors in writing the manuscript. YDW and WYY received, investigated and verified patient data. GX and XHL performed the histological examination of the kidney. XHL, JW, BZ and WYY carried out the cell function tests, dealt with the data and visualized the figures. CMD and BX wrote the original draft. JFF and CMD wrote, revised and edited the final draft. All authors read and approved final version of manuscript.

\section{Acknowledgements}

We would like to give our sincere appreciation to the reviewers for their helpful comments on this.

\section{References}

1. Humphrey PA, Moch H, Cubilla AL, Ulbright TM, Reuter VE. The 2016 WHO Classification of Tumours of the Urinary System and Male Genital Organs-Part B: Prostate and Bladder Tumours. Eur Urol. 2016; 70:106-19.

2. Low G, Huang G, Fu W, Moloo Z, Girgis S. Review of renal cell carcinoma and its common subtypes in radiology.World J Radiol. 2016; 8:484-500.

3. Shingarev R, Jaimes EA. Renal cell carcinoma: new insights and challenges for a clinician scientist.Am J Physiol Renal Physiol. 2017;313:F145-54.

4. Mahasin SZ, Aloudah N, Al-Surimi K, Alkhateeb SS. Epidemiology profile of renal cell carcinoma: A 10-year patients' experience at King Abdulaziz Medical City, National Guard Health Affairs, Saudi Arabia. Urol Ann. 2018; 10:59-64.

5. Feng J, Guo Y, Li Y, Zeng J, Wang Y, Yang Y, et al. Tumor promoting effects of circRNA_001287 on renal cell carcinoma through miR-144-targeted CEP55. J ExpClin Cancer Res. 2020; 39:269.

6. Choueiri TK, Motzer RJ. Systemic therapy for metastatic renal-cell carcinoma. N Engl J Med. 2017; 376:354-66.

7. Abdul-Ghafar J, Ud Din N, Saadaat R, Ahmad Z. Metastatic renal cell carcinoma to pancreas and gastrointestinal tract: a clinicopathological study of 3 cases and review of literature. BMC Urol. 2021; $21: 84$.

8. Hu Q, Lyon CJ, Fletcher JK, Tang W, Wan M, Hu TY. Extracellular vesicle activities regulating macrophage- and tissue-mediated injury and repair responses. Acta Pharm Sin B. 2021;11:1493-512. 
9. Wu T, Dai Y. Tumor microenvironment and therapeutic response. Cancer Lett.2017;387:61-8.

10. Lin Y, Xu J, Lan H. Tumor-associated macrophages in tumor metastasis: biological roles and clinical therapeutic applications. J HematolOncol. 2019; 12:76.

11. Giusti I, Di Francesco M, D'Ascenzo S, Palmerini M, Macchiarelli G, Carta G, et al. Ovarian cancerderived extracellular vesicles affect normal human fibroblast behavior. Cancer BiolTher. 2018; 19:722-34.

12. Dias F, Teixeira AL, Nogueira I, Morais M, Maia J, Bodo C, et al. Extracellular vesicles enriched in hsamiR-301a-3p and hsa-miR-1293 dynamics in clear cell renal cell carcinoma patients: potential biomarkers of metastatic disease. Cancers (Basel). 2020; 12:1450.

13. Hanahan D, Weinberg RA. Hallmarks of cancer: the next generation. Cell. 2011;1 44:646-74.

14. Jurj A, Zanoaga O, Braicu C, Lazar V, Tomuleasa C, Irimie A, et al. A Comprehensive picture of extracellular vesicles and their contents. Molecular transfer to cancer cells. Cancers (Basel). 2020; $12: 298$.

15. Ma Y, Wu T, Ling C, Yu F, Zhang J, Cao P, et al. M2 macrophage-derived exosomal microRNA-155-5p promotes the immune escape of colon cancer by downregulating ZC3H12B. Mol Ther Oncolytics. $2021 ; 20: 484-98$.

16. Pu J, Xu Z, Nian J, Fang Q, Yang M, Huang Y, et al. M2 macrophage-derived extracellular vesicles facilitate CD8+T cell exhaustion in hepatocellular carcinoma via the miR-21-5p/YOD1/YAP/ $\beta$-catenin pathway. Cell Death Discov. 2021; 7:182.

17. Chang J, Li H, Zhu Z, Mei P, Hu W, Xiong X, et al. microRNA-21-5p from M2 macrophage-derived extracellular vesicles promotes the differentiation and activity of pancreatic cancer stem cells by mediating KLF3. Cell Biol Toxicol. 2021; doi:10.1007/s10565-021-09597-x.

18. Ambros V. The functions of animal microRNAs. Nature.2004;431:350- 355.

19. Wang L, Yang G, Zhao D, Wang J, Bai Y, Peng Q, et al. CD103-positive CSC exosome promotes EMT of clear cell renal cell carcinoma: role of remote MiR-19b-3p. Mol Cancer. 2019; 18:86.

20. Song S, Long M, Yu G, Cheng Y, Yang Q, Liu J, et al. Urinary exosome miR-30c-5p as a biomarker of clear cell renal cell carcinoma that inhibits progression by targeting HSPA5. J Cell Mol Med. 2019; 23:6755-65.

21. Wang X, Wang T, Chen C, Wu Z, Bai P, Li S, et al. Serum exosomal miR-210 as a potential biomarker for clear cell renal cell carcinoma. J Cell Biochem. 2018; doi:10.1002/jcb.27347.

22. Komoll RM, Hu Q, Olarewaju O, Dohlen L, Yuan Q, Xie Y, et al. MicroRNA-342-3p is a potent tumour suppressor in hepatocellular carcinoma. J Hepatol. 2021; 74:122-34.

23. Cui Z, Zhao Y. microRNA-342-3p targets FOXQ1 to suppress the aggressive phenotype of nasopharyngeal carcinoma cells. BMC Cancer. 2019; 19:104.

24. Lan J, Sun L, Xu F, Liu L, Hu F, Song D, et al. M2 Macrophage-derived exosomes promote cell migration and invasion in colon cancer. Cancer Res. 2019; 79:146-58. 
25. Chen H, Zhu D, Zheng Z, Cai Y, Chen Z, Xie W. CEP55 promotes epithelial-mesenchymal transition in renal cell carcinoma through PI3K/AKT/mTOR pathway. Clin Transl Oncol. 2019; 21:939-49.

26. Hughes JR, Parsons JL. The E3 ubiquitin ligase NEDD4L targets OGG1 for ubiquitylation and modulates the cellular DNA damage response. Front Cell Dev Biol. 2020; 8:607060.

27. Ribeiro Franco PI, Rodrigues AP, de Menezes LB, Pacheco Miguel M. Tumor microenvironment components: Allies of cancer progression. Pathol Res Pract. 2020; 216:152729.

28. Svoronos AA, Engelman DM, Slack FJ. OncomiR or tumorsuppressor? The duplicity of microRNAs in cancer. CancerRes. 2016; 76:3666-70

29. Zhang S, Liu L, Lv Z, Li Q, Gong W, Wu H. MicroRNA-342-3p inhibits the proliferation, migration, and invasion of osteosarcoma cells by targeting astrocyte-elevated gene-1 (AEG-1). Oncol Res. 2017; 25:1505-15.

30. Xie X, Liu H, Wang M, Ding F, Xiao H, Hu F, et al. miR-342-3p targets RAP2B to suppress proliferation and invasion of non-small cell lung cancer cells. Tumour Biol. 2015; 36:5031-8.

31. Wang S, Ma F, Tang Z, Wu X, Cai Q, Zhang M, et al. Long non-coding RNA H19 regulates FOXM1 expression by competitively binding endogenous miR-342-3p in gallbladder cancer. J Exp Clin Cancer Res. 2016; 35:160.

32. He J, He J, Min L, He Y, Guan H, Wang J, et al. Extracellular vesicles transmitted miR-31-5p promotes sorafenib resistance by targeting MLH1 in renal cell carcinoma. Int J Cancer. 2020; 146:1052-63.

33. Wang X, Duan J, Fu W, Yin Z, Sheng J, Lei Z, et al. Decreased expression of NEDD4L contributes to NSCLC progression and metastasis. Biochem Biophys Res Commun. 2019; 513:398-404.

34. Tanksley JP, Chen X, Coffey RJ. NEDD4L is downregulated in colorectal cancer and inhibits canonical WNT signaling. PLoS One. 2013; 8:e81514.

35. Verma N, Müller AK, Kothari C, Panayotopoulou E, Kedan A, Selitrennik M, et al. Targeting of PYK2 synergizes with EGFR antagonists in basal-like TNBC and circumvents HER3-associated resistance via the NEDD4-NDRG1 axis. Cancer Res. 2017; 77:86-99.

36. Takeuchi T, Adachi Y, Nagayama T, Furihata M. Nedd4L modulates the transcription of metalloproteinase- 1 and -13 genes to increase the invasive activity of gallbladder cancer. Int J Exp Pathol. 2011; 92:79-86.

37. Zhao H, Zhang J, Fu X, Mao D, Qi X,Liang S, et al. Integrated bioinformatics analysis of the NEDD4 family reveals a prognostic value of NEDD4L in clear-cell renal cell cancer. Peer J. 2021; 9:e11880.

38. Qu MH, Han C, Srivastava AK, Cui T, Zou N, Gao Z, et al. miR-93 promotes TGF- $\beta$-induced epithelial-tomesenchymal transition through downregulation of NEDD4L in lung cancer cells. Tumour Biol. 2016; 37:5645-51.

39. Jarome TJ, Devulapalli RK. The Ubiquitin-proteasome system and memory: moving beyond protein degradation. Neuroscientist. 2018;24:639-651.

40. Donovan P, Poronnik P. Nedd4 and Nedd4-2: ubiquitin ligases at work in the neuron. Int J Biochem Cell Biol. 2013; 45:706-10. 
41. Goel P, Manning JA, Kumar S. NEDD4-2 (NEDD4L): The ubiquitin ligase for multiple membrane proteins. Gene. 2015; 557:1-10

42. Kiavue N, Cabel L, Melaabi S, Bataillon G, Callens C, Lerebours F, et al. ERBB3 mutations in cancer: Biological aspects, prevalence and therapeutics. Oncogene. 2020; 39:487-502

43. Zachari M, Ganley IG. The mammalian ULK1 complex and autophagy initiation. Essays Biochem. 2017; 61:585-96.

44. Li TY, Sun Y, Liang Y, Liu Q, Shi Y, Zhang C, et al. ULK1/2 constitute a bifurcate node controlling glucose metabolic fluxes in addition to autophagy. Mol Cell. 2016; 62:359-70

45. Yan SM, Liu L, Gu WY, Huang LY, Yang Y, Huang YH, et al. CEP55 Positively Affects Tumorigenesis of Esophageal Squamous Cell Carcinoma and Is Correlated with Poor Prognosis. J Oncol. 2021; 2021:8890715.

46. Li F, Jin D, Tang C, Gao D. CEP55 promotes cell proliferation and inhibits apoptosis via the $\mathrm{PI3K} / \mathrm{Akt} / \mathrm{p} 21$ signaling pathway in human glioma U251 cells. Oncol Lett. 2018; 15:4789-96.

47. Chen H, Zhu D, Zheng Z, Cai Y, Chen Z, Xie W. CEP55 promotes epithelial-mesenchymal transition in renal cell carcinoma through PI3K/AKT/mTOR pathway. Clin Transl Oncol. 2019; 21:939-49.

48. Guo H, German P, Bai S, Barnes S, Guo W, Qi X, et al. The PI3K/AKT pathway and renal cell carcinoma. J Genet Genomics. 2015; 42:343-53.

49. Lian JH, Wang WH, Wang JQ, Zhang YH, Li Y. MicroRNA-122 promotes proliferation, invasion and migration of renal cell carcinoma cells through the PI3K/Akt signaling pathway. Asian Pac J Cancer Prev. 2013; 14:5017-21.

50. Xu X, Wu J, Li S, Hu Z, Xu X, Zhu Y, et al. Downregulation of microRNA-182-5p contributes to renal cell carcinoma proliferation via activating the AKT/FOXO3a signaling pathway. Mol Cancer. 2014;13:109.

51. Akbani R, Ng PK, Werner HM, Shahmoradgoli M, Zhang F, Ju Z, et al. A pan-cancer proteomic perspective on The Cancer Genome Atlas. Nat Commun. 2014; 5:3887.

\section{Figures}



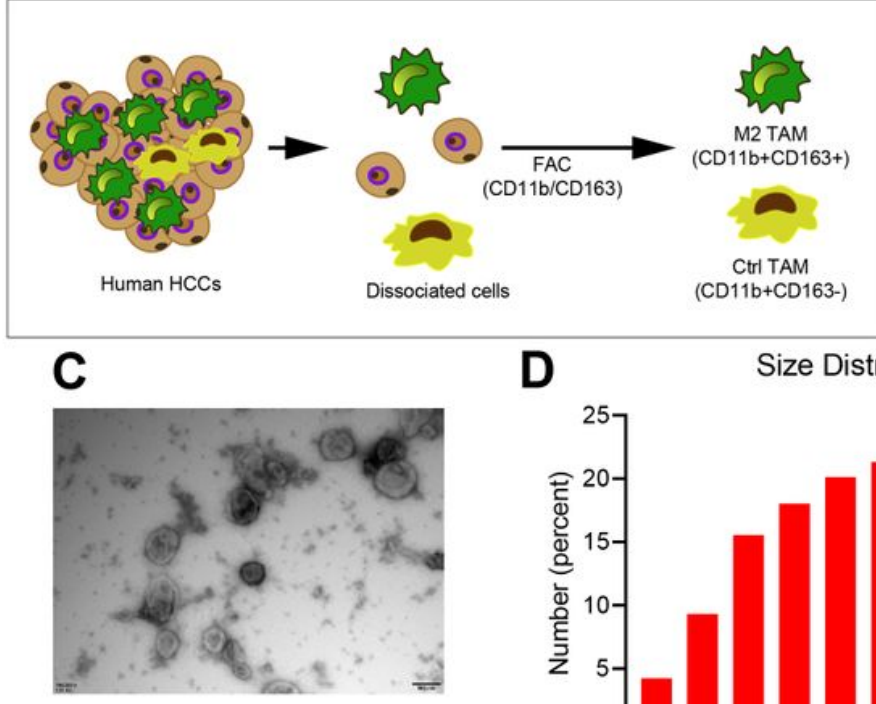

D

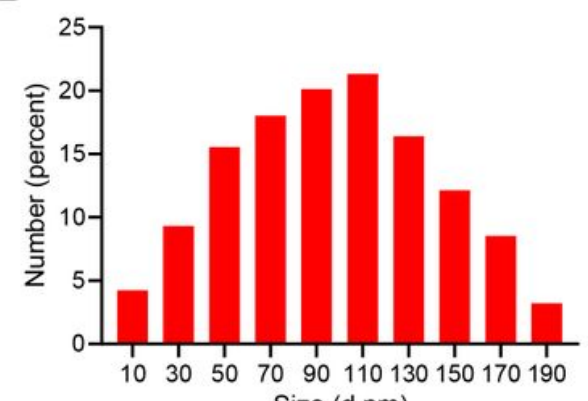

CD68

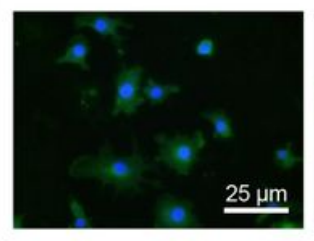

CD163

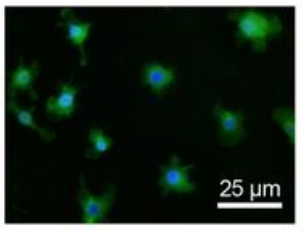

CD206

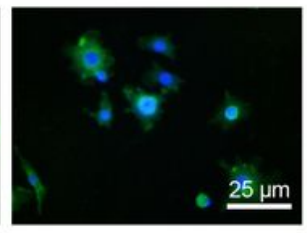

E

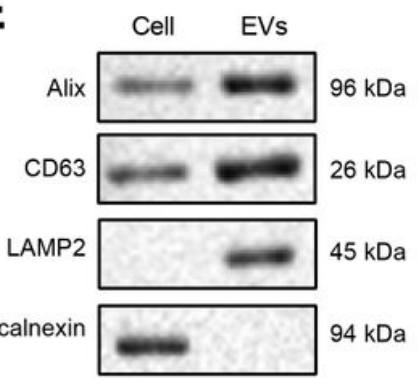

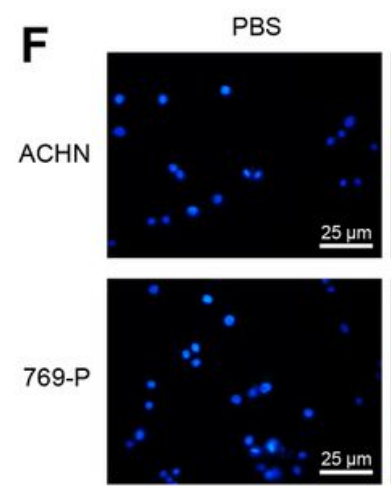

PKH67-EVs

Size (d.nm)
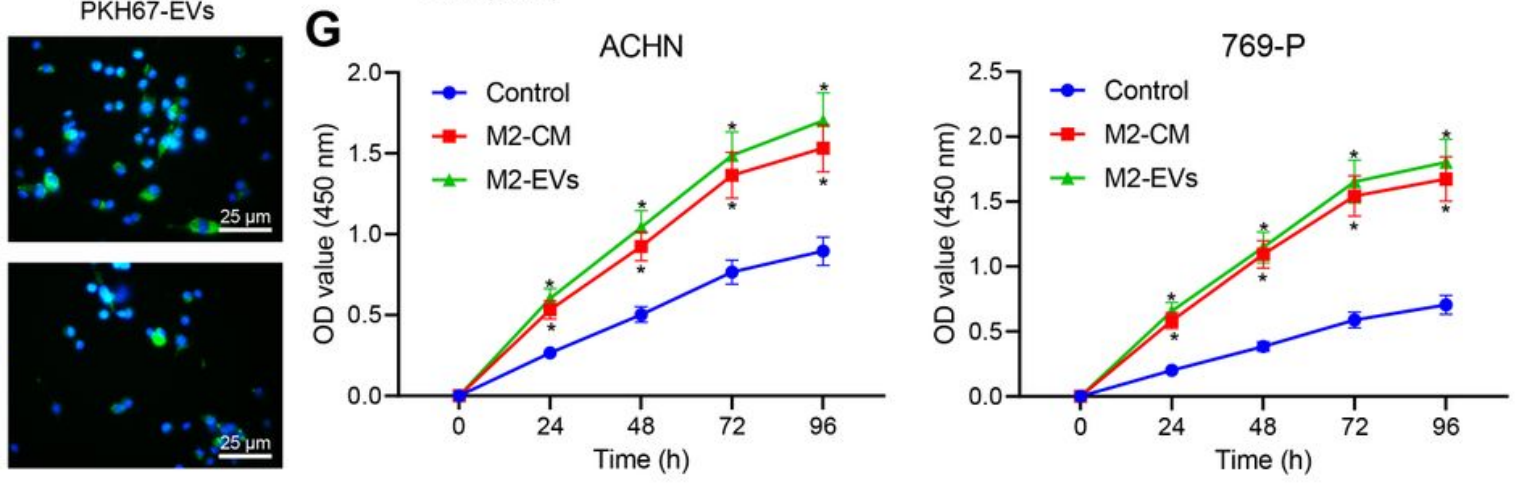

H

H Control

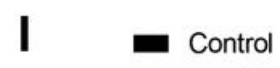

口 M2-CM
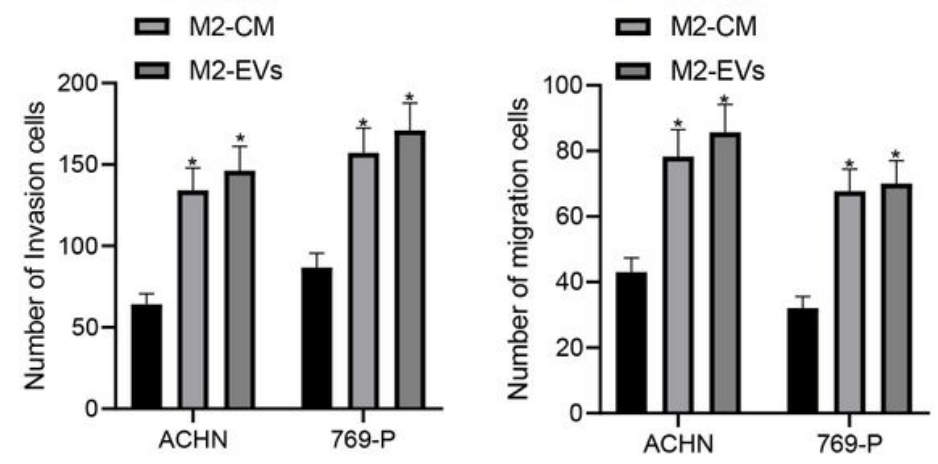

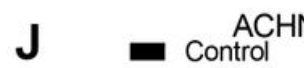

口 M2-CM
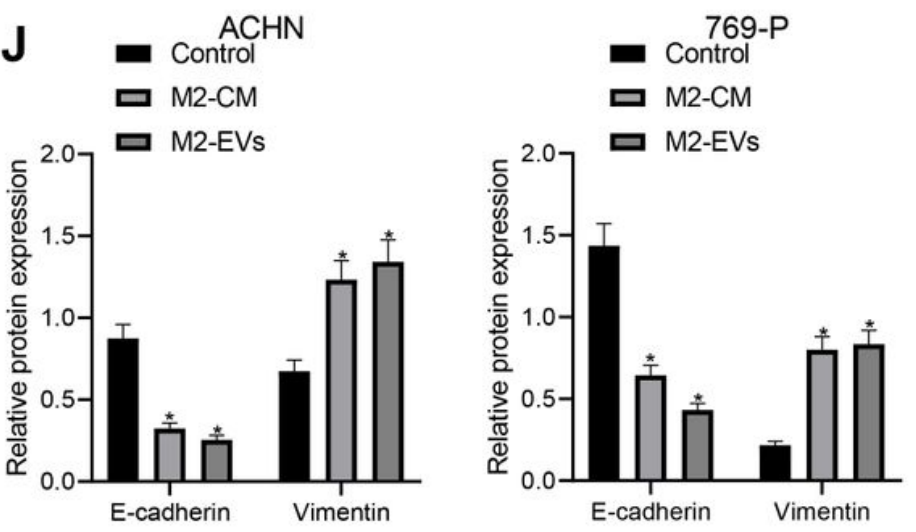

\section{Figure 1}

M2-EVs promote proliferation, migration and invasion of RCC cells

A: Isolation and purification of M2 TAMs from RCC tissues; B: Immunofluorescence staining of M2 TAMs

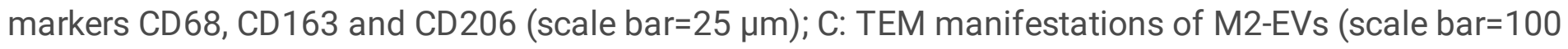
$\mathrm{nm}$ ); D: Particle size distribution of M2-EVs; E: Western blot analysis for EVs surface markers including Hsp70, CD63, LAMP2 and calnexin; F: Internalization of PKH67-labeled EVs by RCC cells (scale bar=25 $\mu \mathrm{m})$; G: Cellular proliferation activities of RCC cells co-cultured with M2-CM or M2-EVs by CCK-8 assay; $\mathrm{H}$ - 
I: Migration and invasion abilities of RCC cells co-cultured with M2-CM or M2-EVs by Transwell assay; J: Western blot analysis for EMT-associated proteins including E-cadherin and Vimentin on RCC cells cocultured with M2-CM or M2-EVs. * indicates $P<0.05$ when compared with Control group. Measurement data were expressed in the form of Mean \pm standard deviation. Data of the two groups were compared using independent sample T test. One-way ANOVA (followed by Tukey post-hoc test) was electively used for data comparison between multiple groups. Data were compared between groups at different time points using repeated measures ANOVA and Tukey post-hoc test.

A

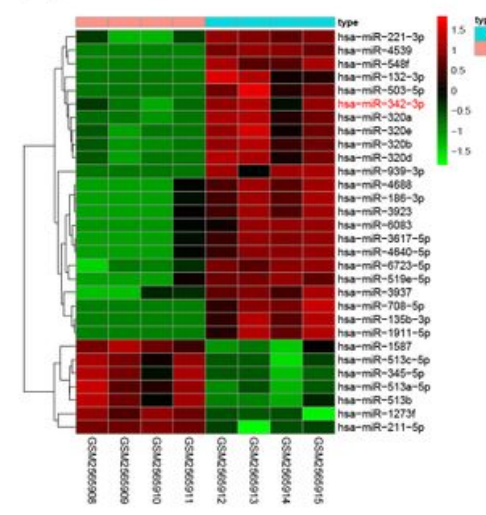

E

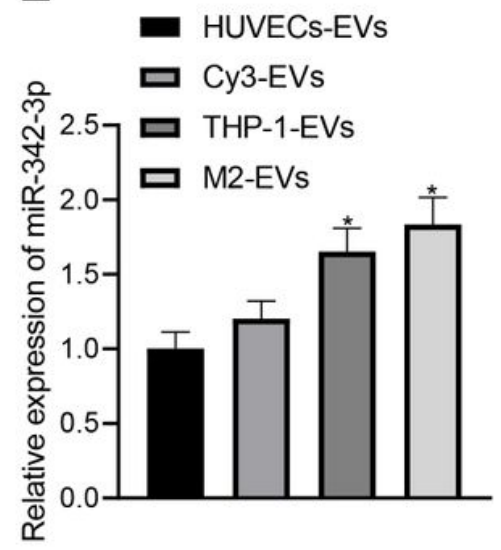

B

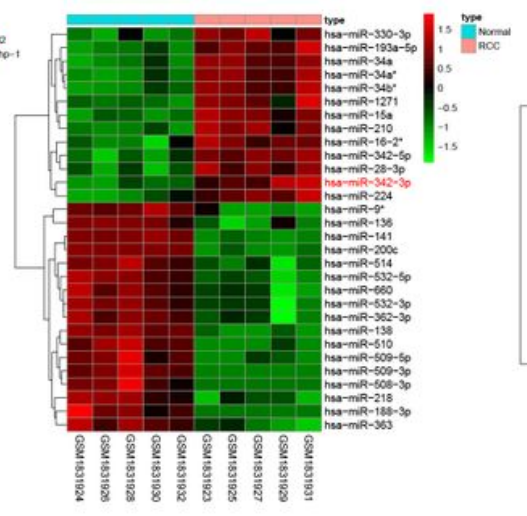

$\mathbf{F}$

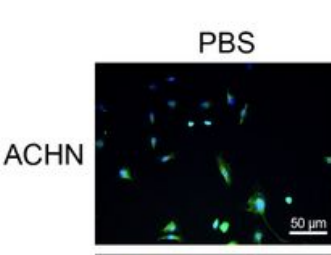

769-P

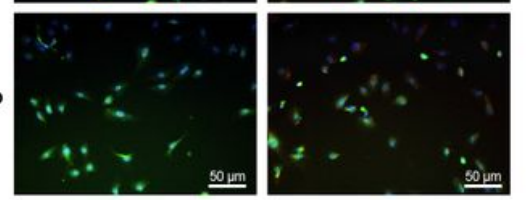

C

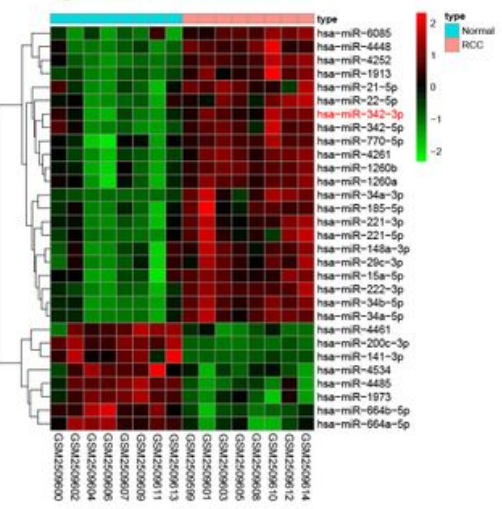

G $\quad A C H N$

- Control

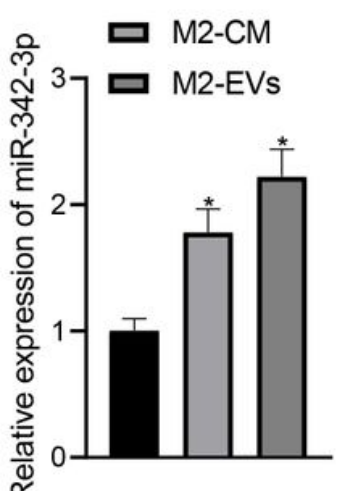

D

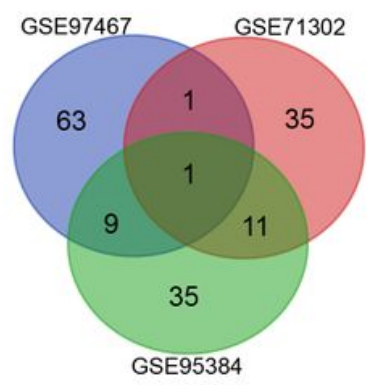

769-P

- Control

ㅁ2-CM

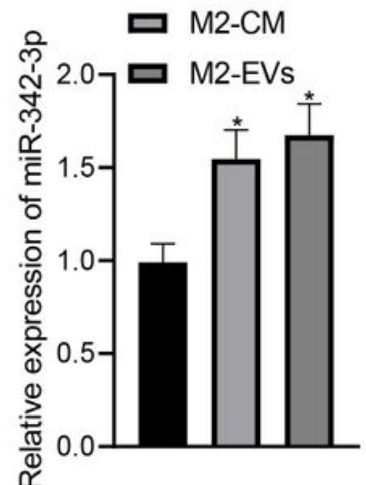

Figure 2

M2-EVs delivers miR-342-3p to RCC cells

A: Heatmap of the top 30 miRNAs differentially expressed between the monocyte THP-1-derived EVs $(n=4)$ and M2-EVs $(n=4)$ samples in GSE97467; B: Heatmap of the top 30 miRNAs differentially expressed between normal kidney $(n=5)$ and RCC tissue $(n=5)$ samples in GSE71302; C: Heatmap of the top 30 miRNAs differentially expressed between normal kidney $(n=8)$ and RCC tissue $(n=8)$ samples in GSE95384 database; D: Venn diagram showing the overlap of up-regulated miRNAs in the three miRNA datasets; E: RT-qPCR assay for miR-342-3p expression in various EVs obtained from different cells (* indicates $P<0.05$, compared to the HUVECs-EVs group); F: Internalization of M2-EVs carrying Cy3-miR342-3p by RCC cells (scale bar $=25 \mu \mathrm{m}$ ) (Green: cytoskeleton by Phalloidin; Blue: nucleus by DAPI); G: RTqPCR assay for miR-342-3p expression in RCC cells co-cultured with M2-CM or M2-EVs. * indicates 
$P<0.05$, compared with control group. Measurement data were expressed in the form of Mean \pm standard deviation. One-way ANOVA (followed by Tukey post-hoc test) was electively used for data comparison between multiple groups.
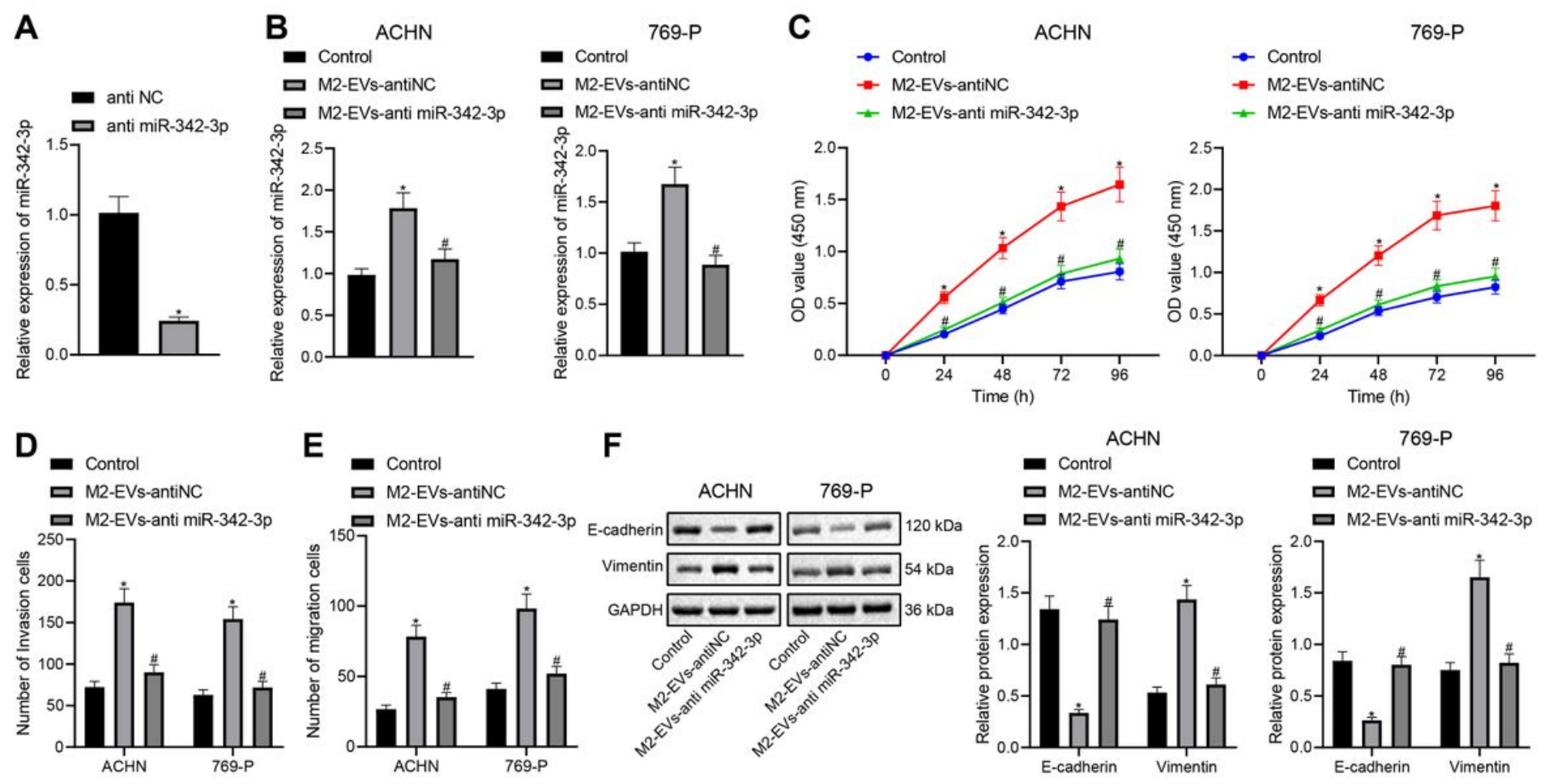

Figure 3

M2-EVs-miR-342-3p promotes proliferation, invasion and migration of RCC cells

A: RT-qPCR assay for miR-342-3p expression in M2-EVs after transfection of anti-NC or anti-miR-342-3p on M2 TAMs; B: RT-qPCR assay for miR-342-3p expression in RCC cells co-cultured with M2-EVs-anti-NC or M2-EVs-anti-miR-342-3p; C-E: Abilities of proliferation, migration and invasion on RCC cells in each treatment group by CCK-8 and Transwell assays; F: Expression of E-cadherin and Vimentin proteins on RCC cells in each treatment group by Western blot. * indicates $P<0.05$, compared to anti NC or Control, \# represents $P<0.05$, compared to M2-EVs-anti NC. Measurement data were expressed in the form of Mean \pm standard deviation. Data of the two groups were compared using independent sample T test. One-way ANOVA (followed by Tukey post-hoc test) was electively used for data comparison between multiple groups. Data were compared between groups at different time points using repeated measures ANOVA and Tukey post-hoc test. 
A

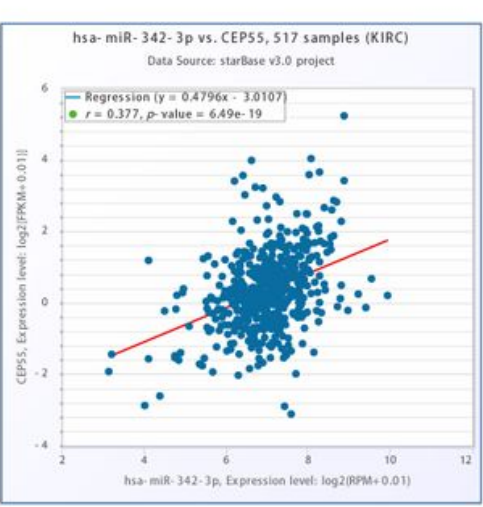

D

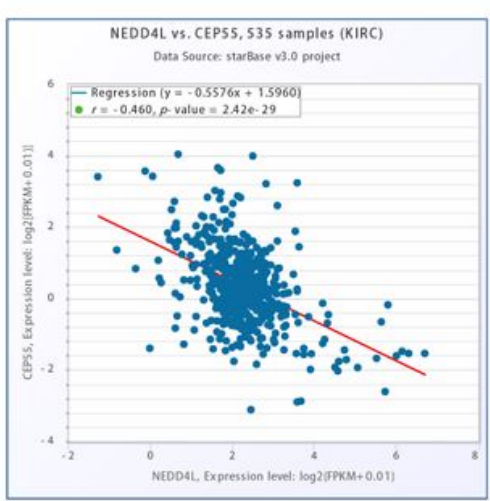

G

G $\operatorname{mimic}_{\text {miR-342-3p mimic }}{ }^{\mathrm{ACHN}}$

口 inhibitor NC

$c^{2.5}$ 口 miR-342-3p inhibitor

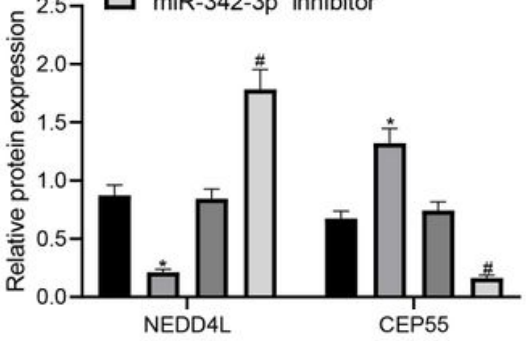

B

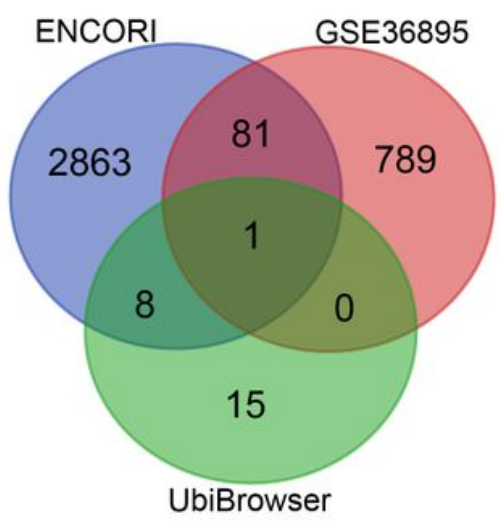

E

NEDD4L WT 5' ccugcccuggcuggcaUGUGAGa 3' miR-342-3p 3' ugcccacgcuaaagacACACÚCu 5' NEDD4LNUT 5' ccugcccuggcuggcaACACGCa 3'

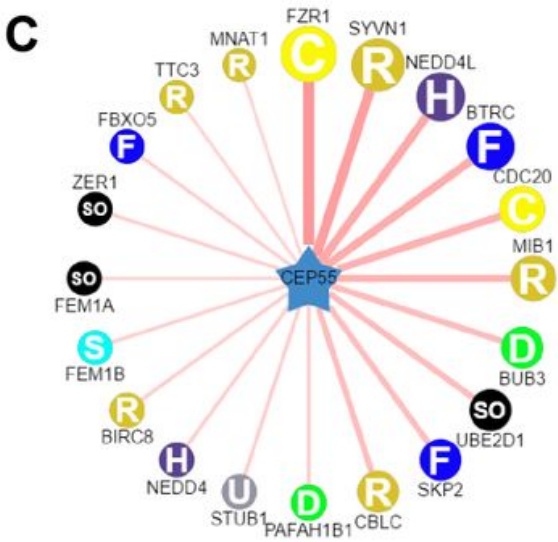

$\mathbf{F}$

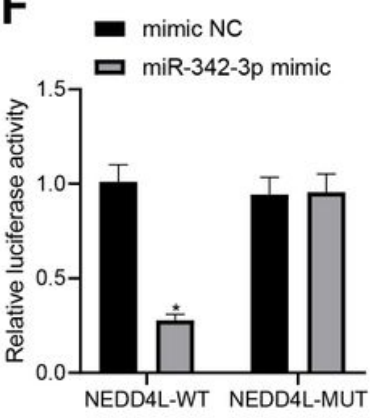

769-P
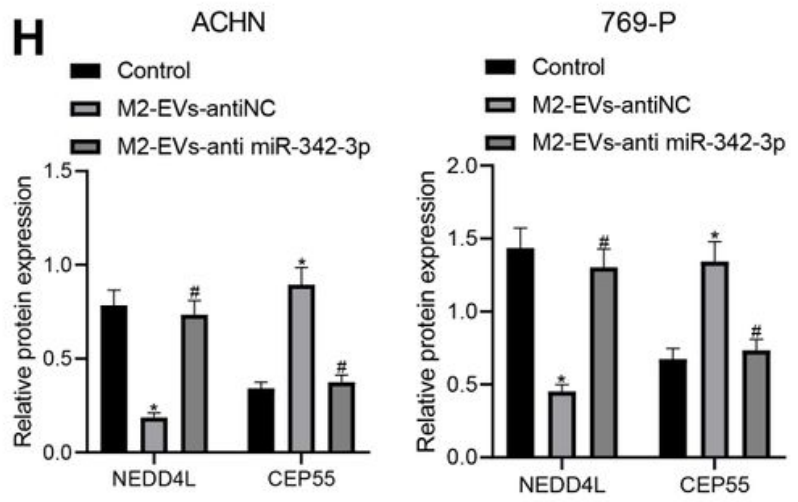

Figure 4

miR-342-3p modulates CEP55 expression by suppressing NEDD4L

A: ENCORI database retrieval showing the correlation between miR-342-3p and CEP55 in RCC; B: Venn diagram showing the overlapped genes from putative targets for miR-342-3p on ENCORI, differentially down-regulated genes in GSE36895 and E3 ubiquitin ligase for CEP55 on UbiBrowser; C: Predicted network diagram of E3 ubiquitin ligases of modulating CEP55 on UbiBrowser; D: Correlation between miR342-3p and CEP55 in RCC on ENCORI; E: Putative miR-342-3p binding sites on NEDD4L on Starbase (http://starbase.sysu.edu.cn/) website; F: Dual luciferase reporter gene assay for HEK293 cells cotransfected by NEDD4L-WT or NEDD4L-MUT with mimic NC or miR-342-3p minic plasmids; G: Protein levels of NEDD4L and CEP55 in RCC cells treated with mimic NC, miR-342-3p mimic, inhibitor NC or miR342-3p inhibitor by Western blot; $\mathrm{H}$ : Protein levels of NEDD4L and CEP55 in RCC cells treated with M2EVs-anti NC or M2-EVs-anti miR-342-3p by Western blot. * indicates $P<0.05$, compared to the mimic NC 
group or control group; \# represents $P<0.05$, compared to inhibitor NC group or M2-EVs-anti NC group. Measurement data were expressed in the form of Mean \pm standard deviation. Data of the two groups were compared using independent sample T test. One-way ANOVA (followed by Tukey post-hoc test) was electively used for data comparison between multiple groups.
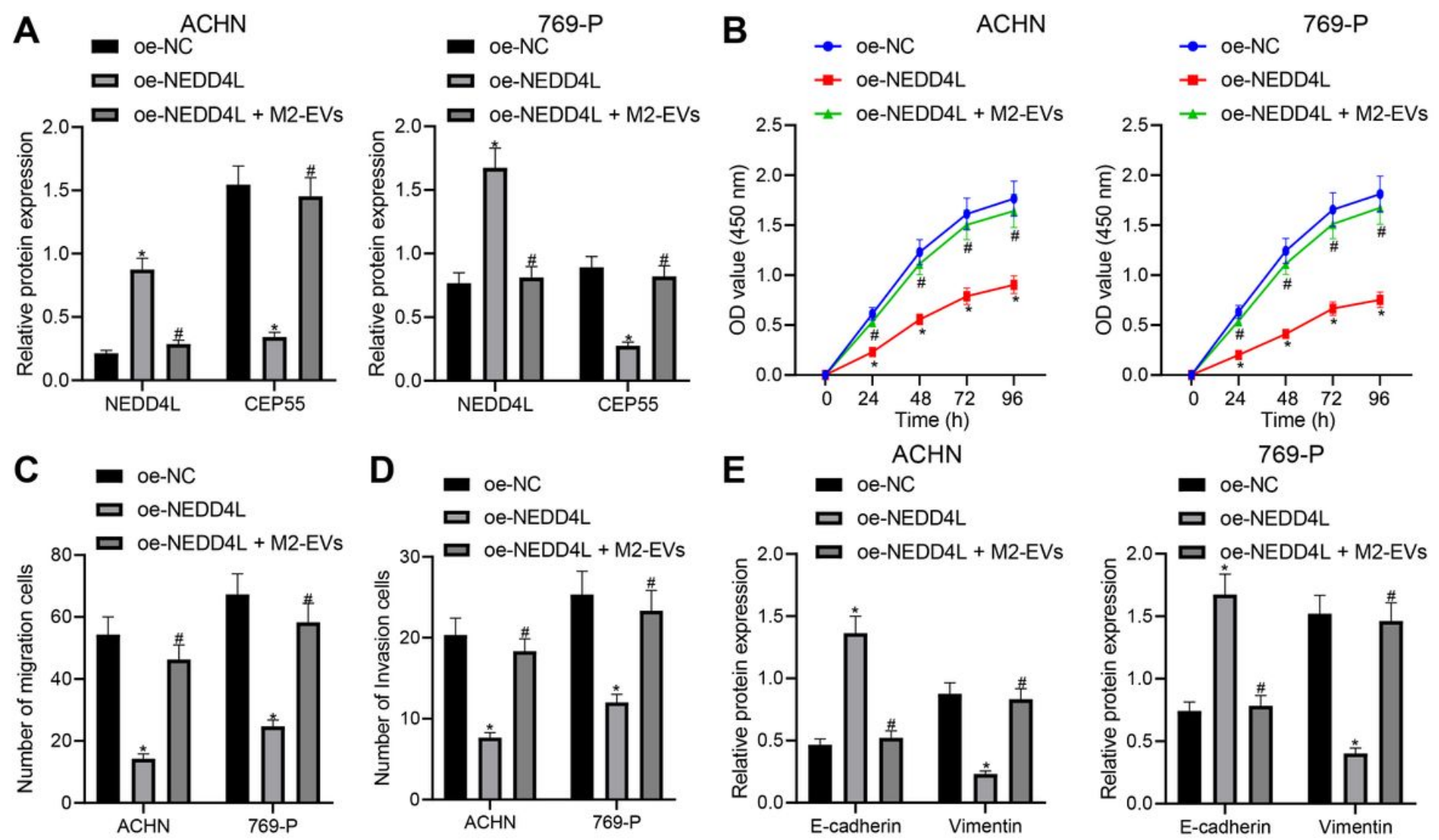

769-P

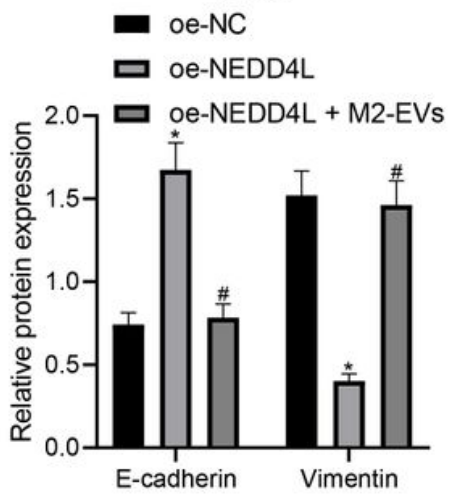

\section{Figure 5}

M2-EVs-miR-342-3p functions on proliferation, migration and invasion of RCC cells via regulating NEDD4L/CEP55 axis

A: Western blot analysis for NEDD4L and CEP55 in RCC cells treated with oe-NEDD4L or oe-NEDD4L+M2EVs; B: RCC cell viability for each treatment group by CCK-8 assay; C-D: RCC cell migration and invasion abilities for each treatment group by Transwell assay; E: Western blot analysis for EMT markers Ecadherin and Vimentin expression in each treatment group of RCC cells. * indicates $P<0.05$, compared to the oe-NC group; \# represents $P<0.05$, compared to the oe-NEDD4L group. Measurement data were expressed in the form of Mean \pm standard deviation. Data of the two groups were compared using independent sample T test. One-way ANOVA (followed by Tukey post-hoc test) was electively used for data comparison between multiple groups. Data were compared between groups at different time points using repeated measures ANOVA and Tukey post-hoc test. 
A

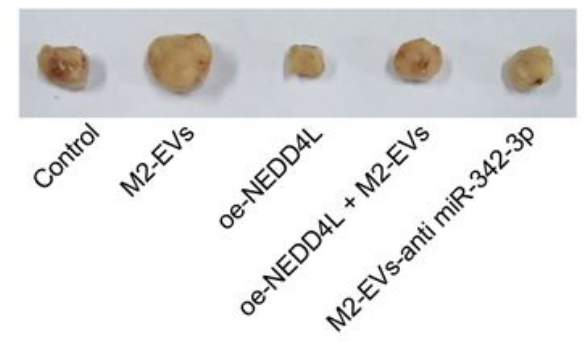

D

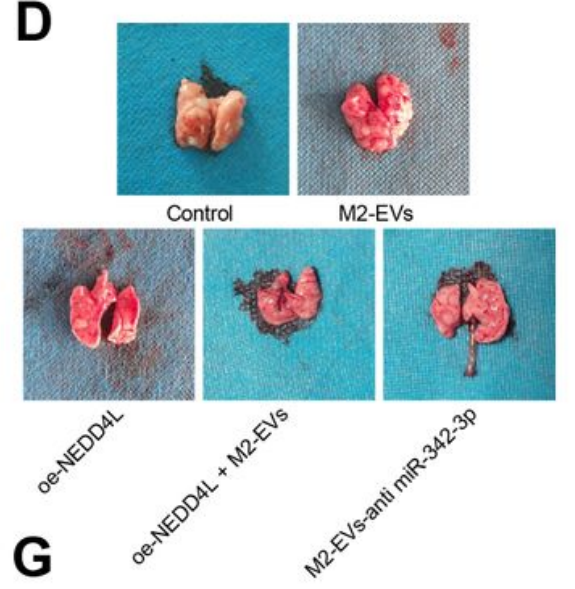

B

$$
\text { - Control }
$$$$
\text { - M2-EVs }
$$$$
\text { - oe-NEDD4L }
$$$$
\text { * oe-NEDD4L + M2-EVs }
$$

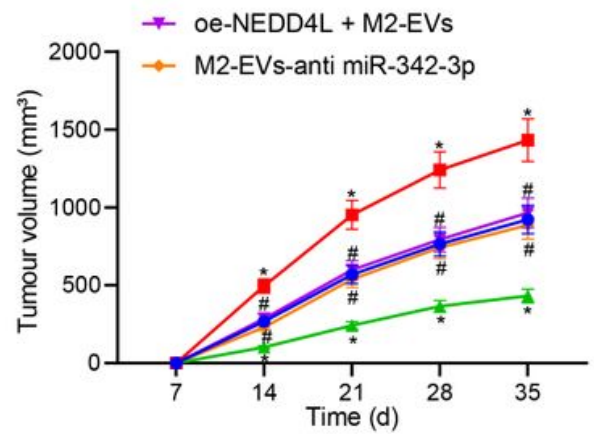

E
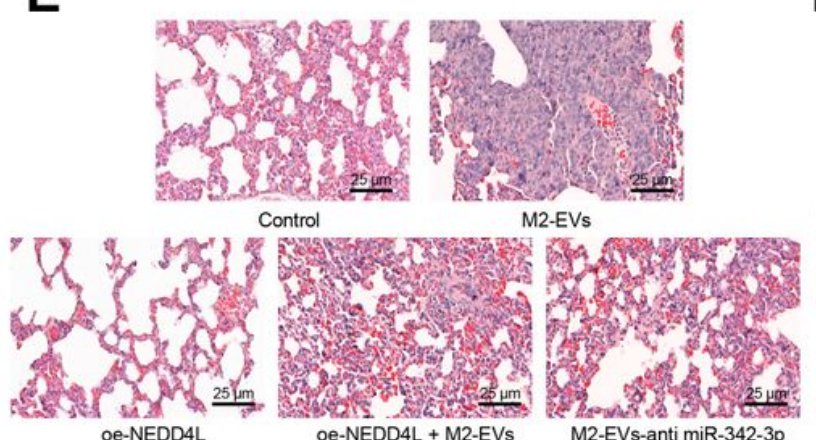

25 m oe-NEDD4L
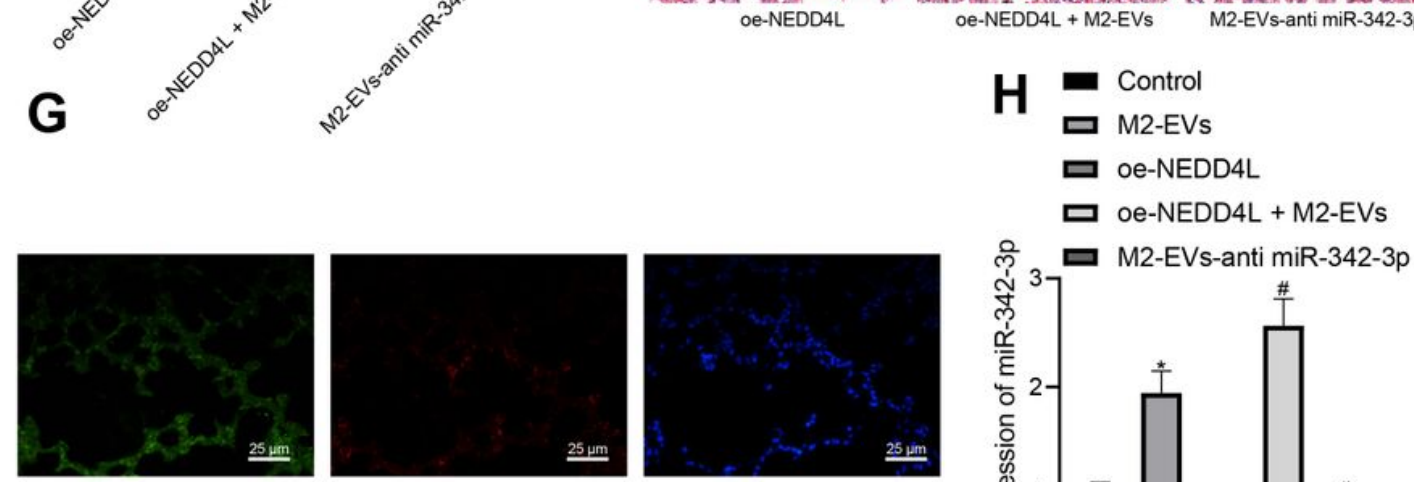

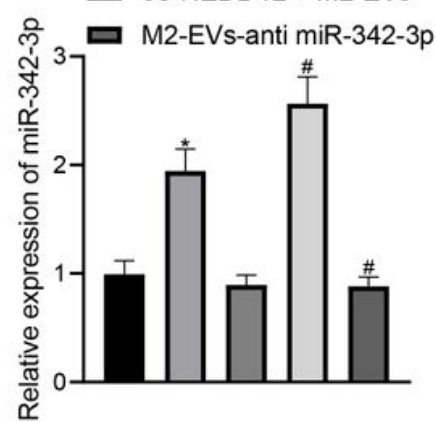

\section{Figure 6}

M2-EVs-miR-342-3p potentiates tumorigenesis and metastasis of RCC via the NEDD4L/CEP55 axis in vivo

A: Representative images of xenograft tumor in M2-EVs, oe-NEDD4L, oe-NEDD4L+M2-EVs, and M2-EVsanti miR-342-3p treated nude mice; B: Changes of tumor volumes in each treatment group in xenograft tumor model; C: Tumor weight in each treatment group in xenograft tumor model; D: Representative images of lung metastasis nodules in M2-EVs, oe-NEDD4L, oe-NEDD4L+M2-EVs, and M2-EVs-anti miR342-3p treated nude mice; $\mathrm{E}$ : H\&E staining on lung tissues in each treatment group in nude mouse model of lung metastasis (scale bar= $25 \mu \mathrm{m}$ ); F: Counts of lung metastasis nodules in each treatment group in nude mouse model of lung metastasis; G: Fluorescence images of Dil-labeled EVs (Red) in lung tissue 
(scale bar=25 $\mu \mathrm{m}$ ); H: RT-qPCR assay for miR-342-3p expression in lung tissue in each treatment group in nude mouse model of lung metastasis; I: Western blot analysis for NEDD4L and CEP55 protein expression in lung tissue in each treatment group in nude mouse model of lung metastasis. * indicates $P<0.05$ compared to the Control group, and \# indicates $P<0.05$ compared to the M2-EVs group.

Measurement data were expressed in the form of Mean \pm standard deviation. One-way ANOVA (followed by Tukey post-hoc test) was electively used for data comparison between multiple groups. Data were compared between groups at different time points using repeated measures ANOVA and Tukey post-hoc test. 

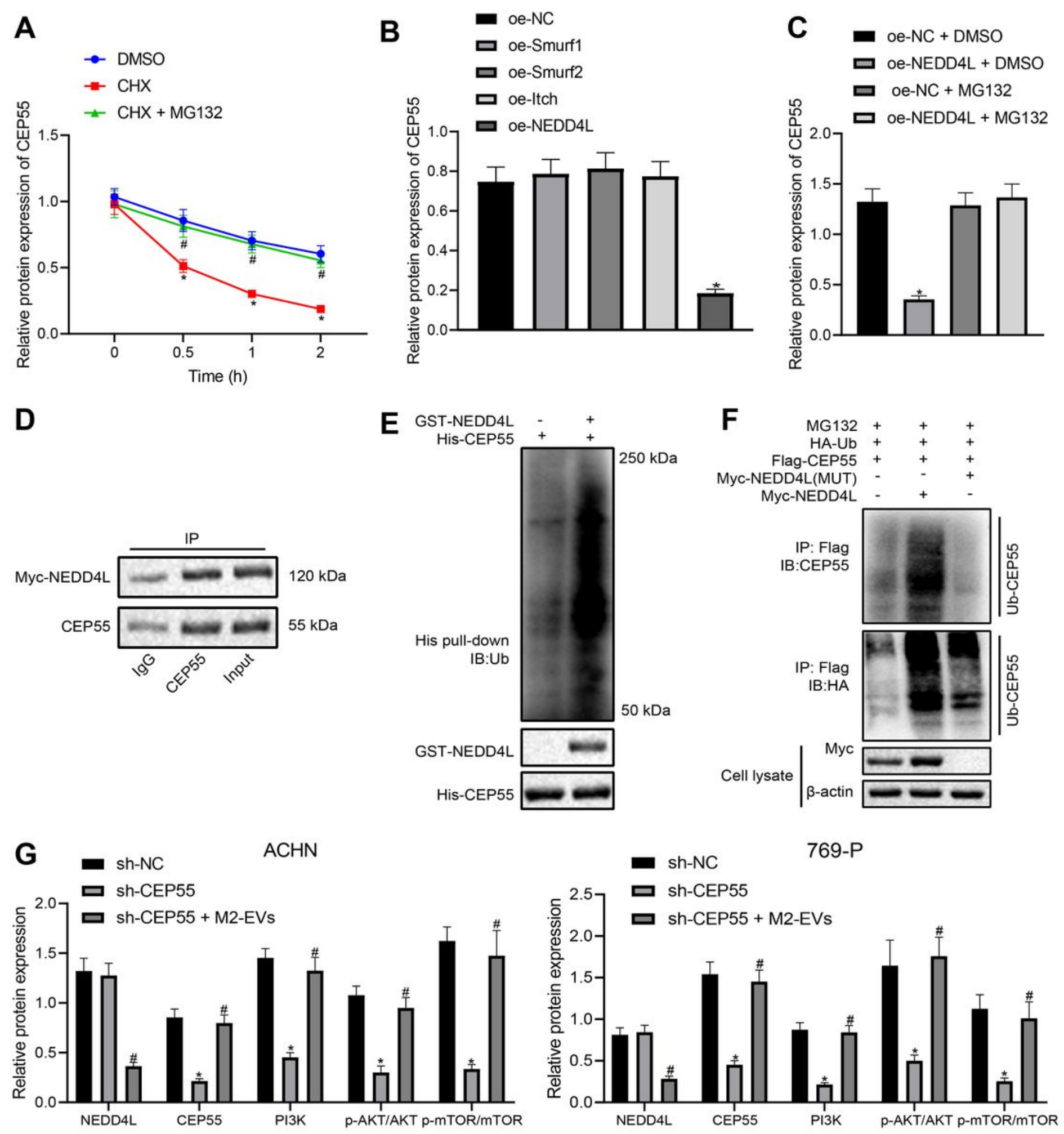

Figure 7

M2-EVs regulates E3 ubiquitin ligase NEDD4L to affect CEP55 ubiquitination and degration and active $\mathrm{PIBK} / \mathrm{AKT} / \mathrm{mTOR}$ signaling pathway

A: Changes of CEP55 level in ACHN cells treated with CHX or CHX-MG132; B: Western blot analysis for CEP55 expression after overexpressing ubiquitin ligases Smurf1, Smurf2, Itch and NEDD4L; C: Western 
blot analysis for CEP55 expression after overexpressing NEDD4L with or without treatment of MG132; D: CO-PI assay to verify the interaction between NEDD4L and CEP55 in ACHN cells (IgG as negative control and Input as positive control); E: In vitro GST-pull down assay to verify the interaction between GSTNEDD4L and His-CEP55 in ACHN cells (GST as negative control and Input as positive control); F: Ubiquitination of CEP55 by NEDD4L as detected by immunoprecipitation (IP) with anti-Flag antibody followed by immunoblotting with anti-CEP55 or anti-HA antibody; G: Protein levels of NEDD4L, CEP55, $\mathrm{PI} 3 \mathrm{~K} / \mathrm{AKT} / \mathrm{mTOR}$ signaling pathway markers (PI3K, p-AKT-Ser473 and p-mTOR-ser2448) in ACHN cells after treatment with sh-CEP55 or sh-CEP55+M2-EVs. * indicates $P<0.05$, compared to the DMSO, oe-NC, oe-NC+DMSO or sh-NC groups; \# indicates $P<0.05$, compared to oe-NC+MG132 or sh-CEP55 groups. Measurement data were expressed in the form of Mean \pm standard deviation. One-way ANOVA (followed by Tukey post-hoc test) was electively used for data comparison between multiple groups. Data were compared between groups at different time points using repeated measures ANOVA and Tukey post-hoc test.
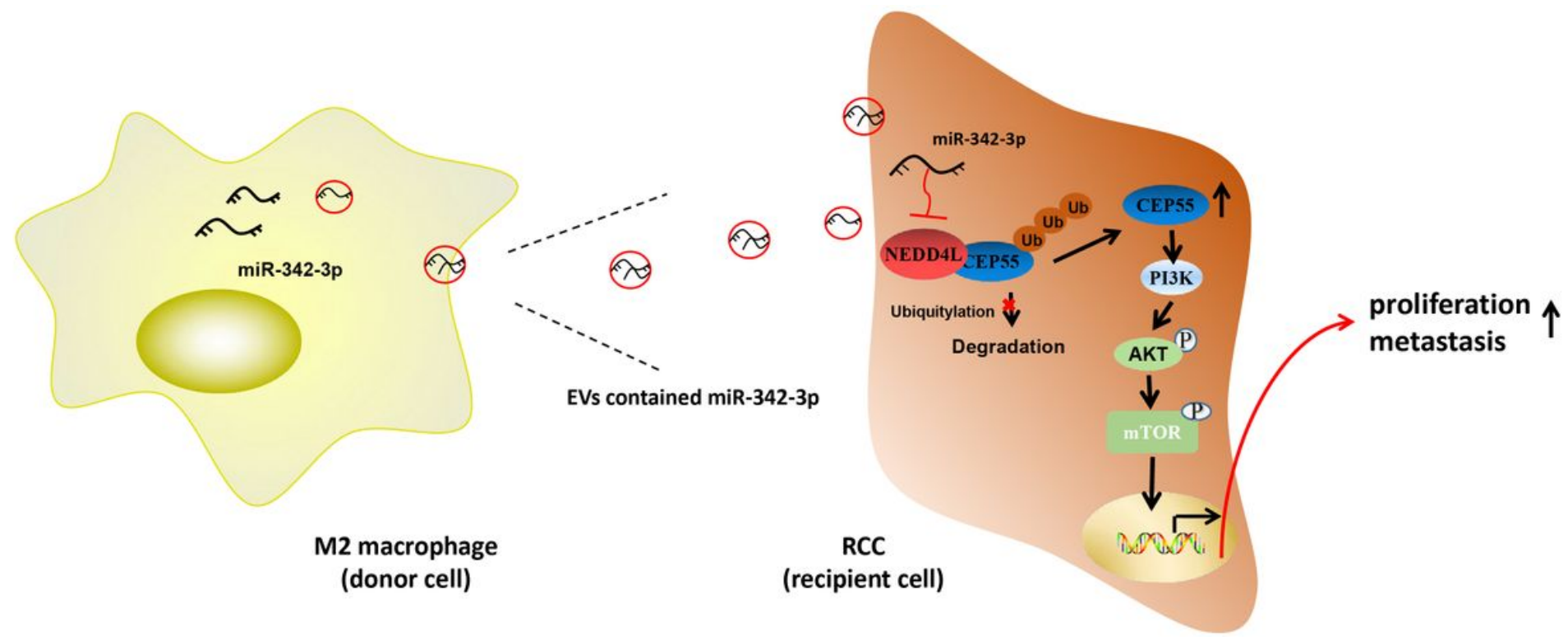

Figure 8

Schematic diagram of the molecular mechanism by which M2-EVs participate in RCC tumorigenesis and progression by delivering miR-342-3p and mediating the NEDD4L/CEP55 axis

\section{Supplementary Files}

This is a list of supplementary files associated with this preprint. Click to download.

- AnnexIStatisticalanalysisofM2.pzfx 